\title{
Revelation of Pivotal Genes Pertinent to Alzheimer's Pathogenesis: A Methodical Evaluation of 32 GEO Datasets
}

\author{
Hema Sree GNS ${ }^{1}$. Saraswathy Ganesan Rajalekshmi ${ }^{1,2}$ - Raghunadha R. Burri ${ }^{3}$
}

Received: 20 August 2021 / Accepted: 18 September 2021 / Published online: 19 October 2021

(c) The Author(s), under exclusive licence to Springer Science+Business Media, LLC, part of Springer Nature 2021

\begin{abstract}
Alzheimer's disease (AD), a dreadful neurodegenerative disorder that affects cognitive and behavioral function in geriatric populations, is characterized by the presence of amyloid deposits and neurofibrillary tangles in brain regions. The International D World Alzheimer Report 2018 noted a global prevalence of 50 million AD cases and forecasted a threefold rise to 139 million by 2050 . Although there exist numerous genetic association studies pertinent to AD in different ethnicities, critical genetic factors and signaling pathways underlying its pathogenesis remain ambiguous. This study was aimed to analyze the genetic data retrieved from 32 Gene Expression Omnibus datasets belonging to diverse ethnic cohorts in order to identify overlapping differentially expressed genes (DEGs). Stringent selection criteria were framed to shortlist appropriate datasets based on false discovery rate (FDR) $p$-value and $\log$ FC, and relevant details of upregulated and downregulated DEGs were retrieved. Among the 32 datasets, only six satisfied the selection criteria. The GEO2R tool was employed to retrieve significant DEGs. Nine common DEGs, i.e., SLC5A3, BDNF, SST, SERPINA3, RTN3, RGS4, NPTX, ENC1 and CRYM were found in more than $60 \%$ of the selected datasets. These DEGs were later subjected to protein-protein interaction analysis with 18 AD-specific literature-derived genes. Among the nine common DEGs, BDNF, SST, SERPINA3, RTN3 and RGS4 exhibited significant interactions with crucial proteins including BACE1, GRIN2B, APP, APOE, COMT, PSEN1, INS, NEP and MAPT. Functional enrichment analysis revealed involvement of these genes in trans-synaptic signaling, chemical transmission, PI3K pathway signaling, receptor-ligand activity and G protein signaling. These processes are interlinked with AD pathways.
\end{abstract}

\section{Keywords BDNF $\cdot \mathrm{SST} \cdot \mathrm{SERPINA} 3 \cdot \mathrm{RTN} 3 \cdot \mathrm{RGS} 4$}

\section{Introduction}

Alzheimer's disease (AD), a progressive irreversible neurodegenerative disorder affecting the elderly, is characterized by dementia and disruption of cognitive functioning. It represents

\section{Highlights}

- Thirty-two AD-specific GEO datasets were screened based on FDR p-value and $\log$ FC

- Nine DEGs were commonly found in more than $60 \%$ of the selected AD datasets

- Five DEGs interacted with BACE1, GRIN2B, APP, APOE, COMT, PSEN1, INS, NEP and MAPT proteins

- BDNF, SST, RTN3 and RSG4 were downregulated, and SERPINA3 was upregulated

- KEGG analysis of DEGs revealed a link with PI3K, G protein and trans-synaptic pathways

Saraswathy Ganesan Rajalekshmi saraswathypradish@gmail.com

Extended author information available on the last page of the article one of the highest unmet medical needs worldwide. The International D World Alzheimer Report 2018 noted a global prevalence of 50 million in 2018 and forecasted a threefold rise in AD cases to 139 million globally by 2050 (International D World Alzheimer Report 2018). In the United States, around 121,000 deaths due to Alzheimer's dementia were reported in 2019. During the coronavirus disease 2019 (COVID-19) pandemic, fatality rates amongst $\mathrm{AD}$ patients increased by 145\% (Alzheimer's disease facts and figures 2021). The Alzheimer's and Related Disorders Society of India (ARDSI) forecasts a huge burden of 6.35 million AD cases across India by 2025 (Kumar et al. 2020).

To date, the US Food and Drug Administration (US-FDA) has approved only four anti-AD drugs, belonging to the following categories: (i) cholinesterase inhibitors: donepezil, rivastigmine and galantamine; and (ii) $\mathrm{N}$-methyl-D-aspartate receptor antagonist: memantine (Alzheimer's Association 2017). The AD treatments are oriented towards nominal symptomatic relief and offer modest clinical effect.

Looking into the pathophysiology, neuropathological evidence shows that $\mathrm{AD}$ is characterized by the presence of 
amyloid beta $(\mathrm{A} \beta)$ plaques and neurofibrillary tangles (NFT) in the hippocampal and cortical regions. Although there are various complex pathophysiological theories explaining the role of numerous genes and proteins in $\mathrm{AD}$ progression, a major role is attributed to presenilin 1 (PSEN1), beta-secretase 1 (BACE1), amyloid precursor protein (APP) and microtubule-associated protein tau (MAPT) proteins (Chouraki and Seshadri 2014). Disruption in regulatory activities such as phosphorylation and dephosphorylation of these proteins result in $\mathrm{AD}$ progression. Notwithstanding the existence of countless genetic evaluations, inconsistencies among various ethnicities contribute to a lacuna in unraveling crucial disease-specific targets. This study was aimed at exploring the major genetic alterations among various microarray datasets to retrieve common differentially expressed genes (DEGs) among various ethnicities, with the hypothesis that overlapping DEGs across different ethnicities might play a definitive role in $\mathrm{AD}$ pathogenesis.

\section{Methodology}

\section{Selection of Datasets}

Microarray datasets pertaining to Alzheimer's disease were retrieved from the Gene Expression Omnibus (GEO) database (Barrett et al. 2013) using the keywords "Alzheimer's disease", "Familial Alzheimer's disease", "Sporadic Alzheimer's disease," "Early onset Alzheimer's disease" and "Late onset Alzheimer's disease". The datasets retrieved through the above search terms were screened through a set of inclusion and exclusion criteria.

\section{Inclusion Criteria}

Datasets satisfying all the following criteria were selected:

- Datasets with controls and AD

- Datasets with expressional arrays

- Datasets describing the diagnostic criteria of $\mathrm{AD}$

- Datasets studied in Homo sapiens

- Datasets with a minimum of two samples in each category, i.e., control and $\mathrm{AD}$

- Datasets with blood/brain samples

\section{Exclusion Criteria}

Datasets with the following criteria were excluded.

- Drug-treated datasets

- Methylation studies

- Datasets with no diagnostic criteria

- Cell line studies

- Datasets from other organisms
- Datasets with no details about controls

- Mutation studies

\section{Gene Expression Analysis}

The selected datasets were preprocessed, curated and analyzed individually for retrieval of differentially expressed genes (DEGs) (both upregulated and downregulated) through the Bioconductor package. The datasets which revealed DEGs with a false discovery rate (FDR) $p$-value (adjusted $p$-value according to Benjamini-Hochberg method) $<0.05$ were selected. These datasets were then subjected to four sets of filtering criteria based on FDR and log fold change (FC): (i) FDR $p$-value $<0.05$ and log $\mathrm{FC}>2$, (ii) FDR $p$-value $<0.05$ and $\log \mathrm{FC}>1.5$, (iii) FDR $p$-value $<0.05$ and $\log \mathrm{FC}>1$ and (iv) FDR $p$-value $<0.01$ and $\log \mathrm{FC}>1$. Based on the above stringent filtering criteria, the datasets possessing the following characteristics were included: (a) datasets satisfying one of the above four criteria, (b) datasets that encompassed both upregulated and downregulated DEGs and (c) $60 \%$ of the datasets showing the aforementioned characteristics (a) and (b) that display a higher degree of common DEGs.

\section{Protein-Protein Interaction (PPI) Analysis}

The common DEGs retrieved from the above step were subjected to PPI analysis with literature-derived genes (LDGs) gathered from the National Center for Biotechnology Information (NCBI) (Brown et al. 2015) pertinent to AD progression through the Search Tool for the Retrieval of Interacting Genes/Proteins (STRING) database (von Mering et al. 2003). The PPI network was visualized through Cytoscape with proteins as nodes and interactions as edges. The proteins exhibiting significant interactions $(70 \%$ confidence score) with LDGs were shortlisted, and the nodes exhibiting node degree $>2$ were selected as $\mathrm{AD}$ targets.

\section{Functional Enrichment Analysis}

The common DEGs retrieved were subjected to functional enrichment analysis to explore their involvement in signaling pathways and physiological functions associated with AD pathogenesis through ClueGO (Bindea et al. 2009) in Cytoscape.

\section{Results}

\section{Selection of Datasets}

A total of 134 GEO datasets derived from studies performed on Homo sapiens were retrieved from NCBI, of which 32 


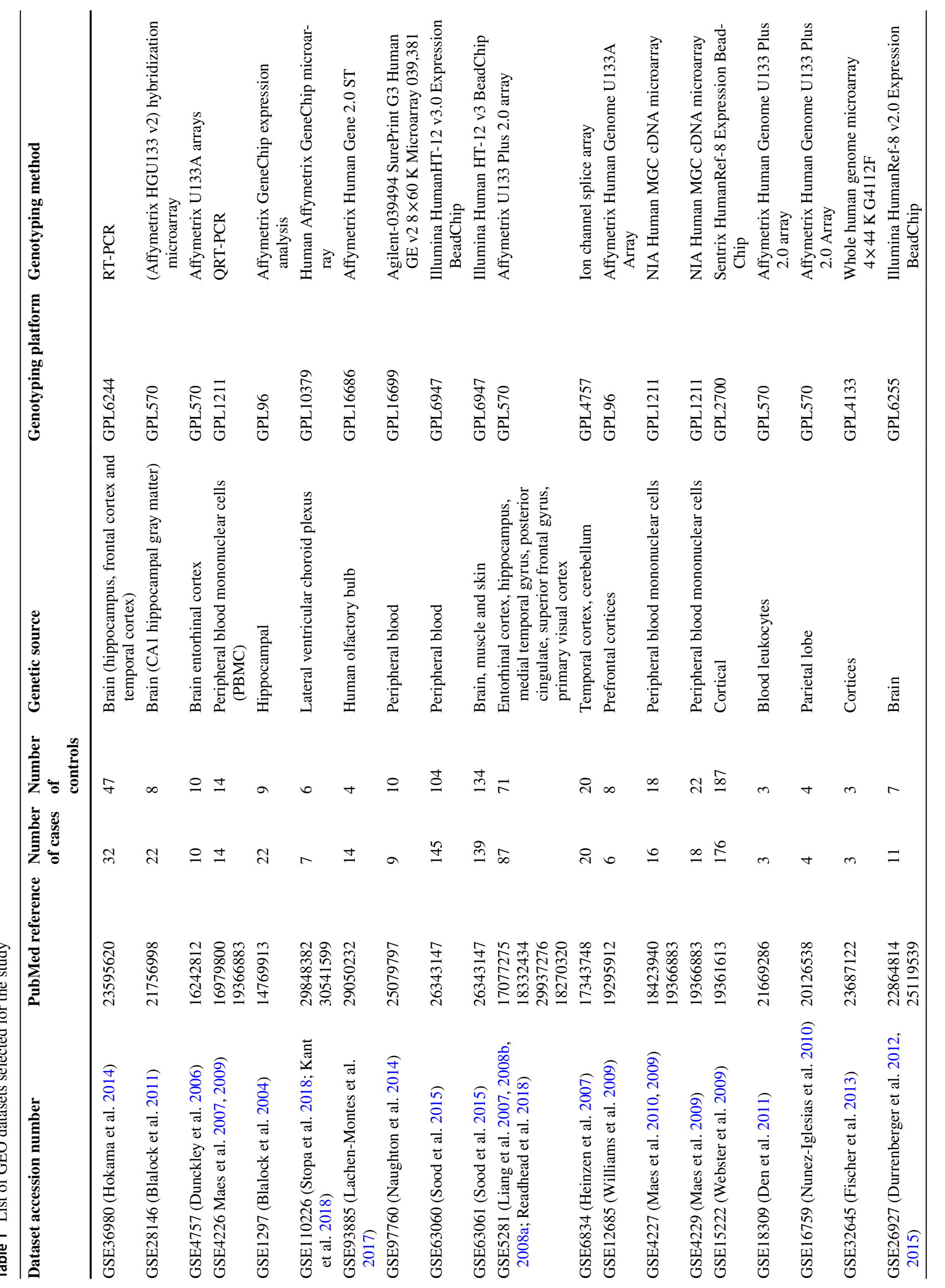




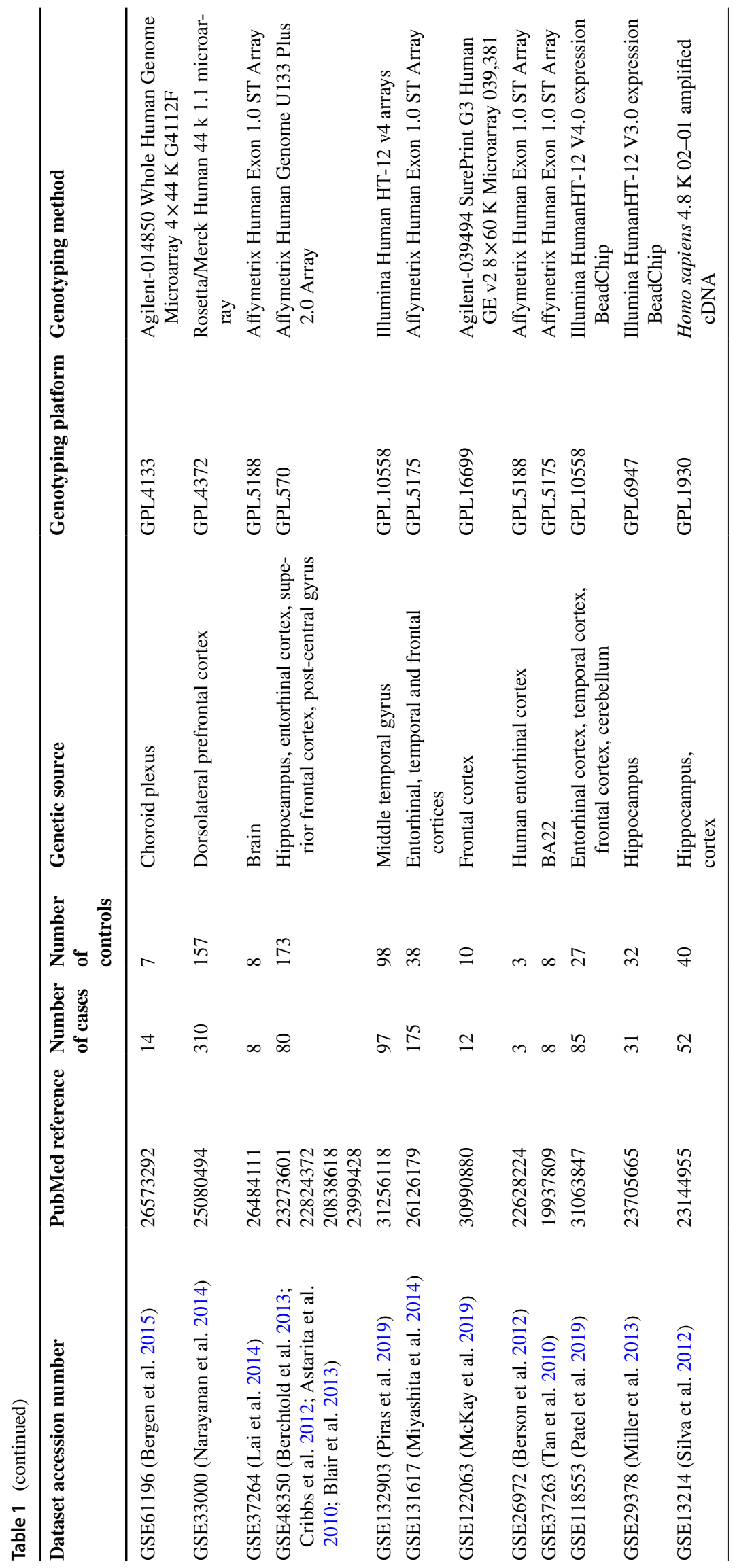


Fig. 1 CONSORT diagram explaining the selection and screening of datasets

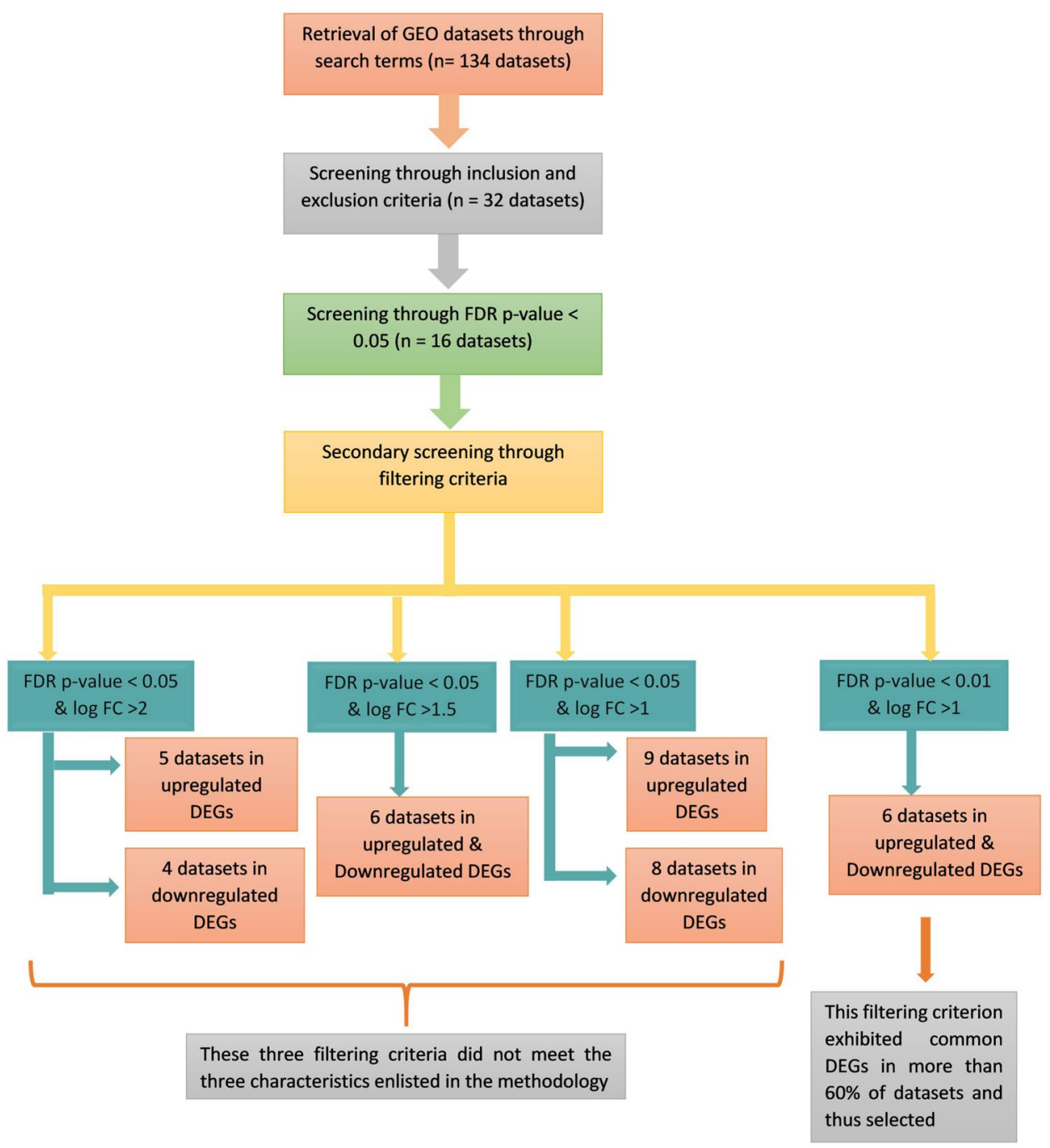

a

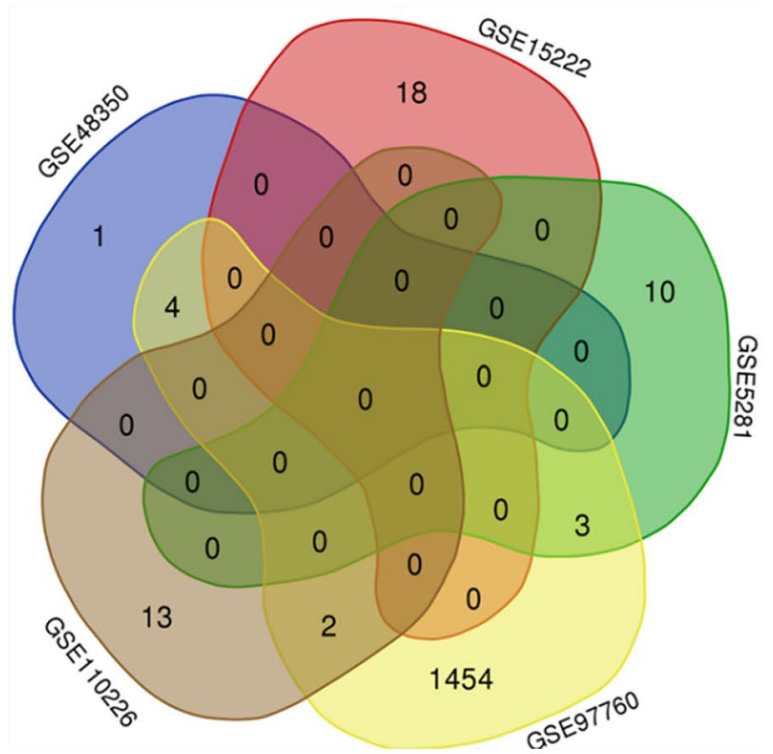

b

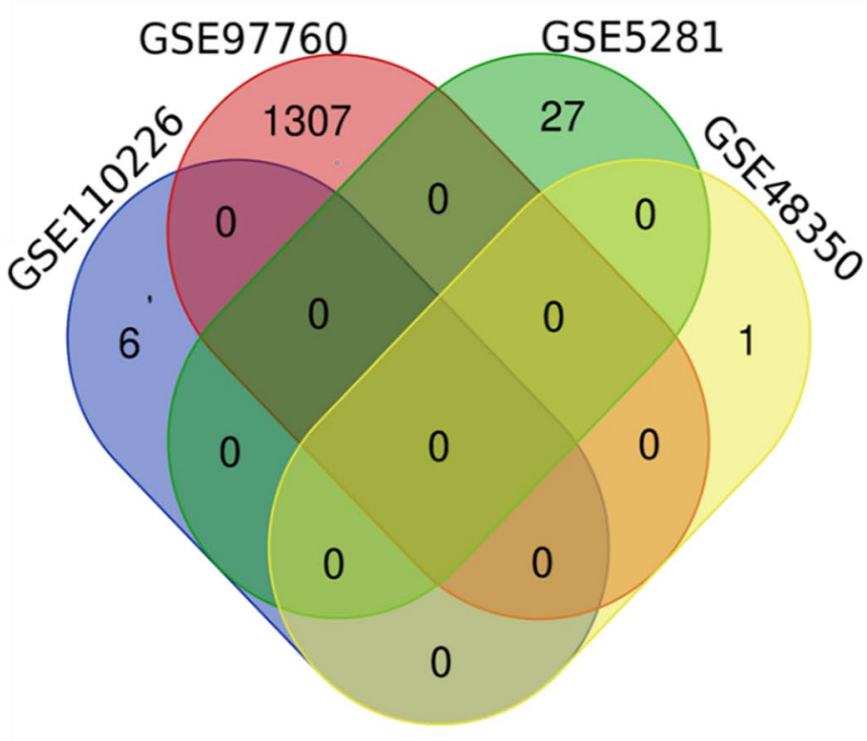

Fig. 2 Venn diagram exhibiting the common upregulated (a) and downregulated (b) DEGs 
Table 2 Number of DEGs obtained through filtering criteria

Dataset accession number

Number

of DEGs

FDR $p$-value $<0.05$ and $\log$ FC $>2$

Upregulated

GSE110226

GSE15222

GSE48350

GSE5281

GSE97760

Downregulated

GSE110226

GSE48350

GSE5281

GSE97760

FDR $p$-value $<0.05$ and $\log$ FC $>1.5$

Upregulated

GSE110226

GSE122063

GSE15222

GSE48350

GSE5281

GSE97760

Downregulated

GSE110226

GSE122063

GSE15222

GSE48350

GSE5281

GSE97760

FDR $p$-value $<0.05$ and $\log$ FC $>1$

Upregulated

GSE110226

GSE131617

GSE132903

GSE15222

GSE29378

GSE48350

GSE5281

GSE63061

GSE97760

Downregulated

GSE110226

GSE122063

GSE132903

GSE15222

GSE48350

GSE5281

GSE63060

GSE97760
Table 2 (continued)

\begin{tabular}{ll}
\hline Dataset accession number & $\begin{array}{l}\text { Number } \\
\text { of DEGs }\end{array}$
\end{tabular}

FDR $p$-value $<0.01$ and $\log$ FC $>1$

Upregulated

GSE122063 386

GSE132903 2

GSE15222 111

GSE48350 11

GSE5281 834

GSE97760 2987

Downregulated

GSE122063 653

GSE132903 28

GSE15222 45

GSE48350 9

GSE5281 1449

GSE97760 1580

datasets were found to satisfy the initial inclusion criteria. Details pertaining to the 32 datasets are presented in Table 1 .

\section{Gene Expression Analysis}

The datasets were analyzed individually through Bioconductor package in R using GEO2R tool (Barrett et al. 2013). Among the 32 datasets, 16 were rejected because they did not exhibit significant FDR $p$-values. The remaining 16 datasets were analyzed based on the four filtering criteria and three characteristics mentioned in the methodology section (Fig. 1).

(i) FDR $p$-value $<0.05$ and $\log$ FC $>2$ :

Out of the 16 qualified datasets, five possessing upregulated DEGs and four with downregulated DEGs (Fig. 2) satisfied this criterion (Tables 2 and 3). Nevertheless, the upregulated DEGs of two datasets of the five displayed overlapping genes, while the downregulated DEGs of the shortlisted datasets did not show common genes. Therefore, this criterion was rejected.

(ii) FDR $p$-value $<0.05$ and $\log \mathrm{FC}>1.5$ :

Among the 16 datasets, only six were found to meet this criterion (Tables 2 and 3). Common DEGs were found in datasets which accounted for $50 \%$ and thus did not meet characteristic (c) mentioned in the methodology section (Fig. 3). Thus, this criterion was also rejected.

(iii) FDR $p$-value $<0.05$ and $\log$ FC $>1$

Among the 16 datasets, this criterion was met by nine datasets with upregulated DEGs and eight datasets with downregulated DEGs (Tables 2 and 3). Also, 
Table 3 List of common DEGs obtained through filtering criteria

Dataset no Common DEGs

FDR $p$-value $<0.05$ and $\log$ FC $>2$

Upregulated

GSE48350 and GSE97760

GSE5281 and GSE97760

GSE110226 and GSE97760

\section{Upregulated}

GSE110226, GSE122063 and GSE97760

GSE122063, GSE5281 and GSE97760

GSE15222, GSE5281 and GSE97760

GSE122063, GSE48350 and GSE97760

GSE5281 and GSE97760

GSE122063 and GSE97760

GSE48350 and GSE97760

GSE15222 and GSE97760

GSE122063 and GSE5281

Downregulated

GSE122063, GSE15222 and GSE5281

GSE110226 and GSE97760

GSE110226 and GSE122063

GSE5281 and GSE97760

GSE15222 and GSE97760

GSE122063 and GSE97760

GSE122063 and GSE5281

GSE122063 and GSE5281

\section{FDR $p$-value $<0.05$ and $\log$ FC $>1$}

\section{Upregulated}

GSE132903, GSE15222, GSE5281 and GSE97760

GSE110226, GSE29378 and GSE97760

GSE15222, GSE5281 and GSE97760

GSE131617, GSE5281 and GSE97760

GSE110226 and GSE97760

GSE110226 and GSE5281

GSE110226 and GSE15222

GSE5281 and GSE97760

GSE15222 and GSE97760

GSE48350 and GSE97760 GSE29378 and GSE97760 GSE15222 and GSE5281 GSE48350 and GSE5281 GSE29378 and GSE5281 GSE132903 and GSE5281 GSE15222 and GSE48350

\section{Downregulated}

GSE110226, GSE122063, GSE5281 and GSE97760 GSE122063, GSE15222, GSE5281 and GSE97760

\author{
SLC25A46, ZNF621, XIST and ANKIB1 \\ RBM33, NEATI and MALATI \\ ILIRLI and SERPINA3 \\ SERPINA3 and ILIRLI \\ NEATI \\ SLC5A3 \\ $X I S T$
}

RGPD5, JPX, ZMYM5, CCDC144A, SNRNP48, ZBED6, SKI, ANKRD36, MECOM, ZDHHC21, UBE3A, RAB18, RBM25, RGPD6, RBM33, RRBP1, SEPT7, GOLIM4, ANKRD12, ZC3H11A, MALAT1 and RANBP2

CCDC66, HMBOXI, ILI8RI and GON4L

SLC25A46 and ANKIB1

RAD51C and F8

SOCS3 and SNX31

RGS4 and SST

SFRP2, TCF21 and HMGCLL1

CTXN3

TSTA3, DUSP4, DCTN1, SLIT3, SEZ6L2, CALY, SNCA, BLVRB, INA, PTPRF, CPNE6, ATP6 and VIG2

NELLI

GPR88, STMN1, RPH3A, DNAH2 and NRIP3

RTN1, BDNF, VSNL1, NMNAT2, RPS4Y1, PTPN3 and MAL2

HSPB3

SLC5A3

SERPINA3

RHOQ and IL6ST

PPA2

ILIRLI, IL4R, IL18RI and C1orf21

SOCS3, MT2A, C10orf54, FBXO32, BACE2, GALNT15 and SLCO4A1

GGPRC5A

HD9, IPW, QKI IL6R, PTPN2, UBE2W, AHNAK, JPX, CASC4, RDX, FAM161A, ZMYM5, SET, FAM120A, SNORA18, BDPI, C5orf56, PPFIBP1, YTHDC2, ELF1, CCDC144A, TAF1D, ZNF713, SNRNP48, SNORD107, SNORD50B, LRRFIP1, ELK4, GRAMDIC, SNORD61, LMO7, SAMHD1, PTBP3, TRIM4, CXCL2, TNPO1, CDK13, ZFP36L1, SEPT8, STAG1, SKI,

TBLIXR1, SNORA I, ANKRD36, CPEB4, MKL2, MBTD1, HCG18, ZNF160, MECOM, PDE4DIP, ZDHHC21, CBX3, TFEB, SKIL, TLE4, IFNAR2, KCNJ16, SLC4A4, KTN1, SAT1, ABLIM1, ZNF28OD, RBMS1, LZTS2, LPP, ATRX, MACF1, PCMTD2, C5orf24, TPP2, SFPQ, ZSCAN30, STAG2, RBM33, RAPH1, SOS2, SNORA40, WHAMMP2, NEAT1, ZNF566, PIK3C2A, NOTCH2NL, LEF1, NEKI, MYH11, SNORD5, ITPR2, SEPT7, PTAR1, FXR1, TUBE1, SGPP2, USP6, FAM198B, ZBTB1, SNORA8, TP53INP1, SNORD84, FAM185A, NFATC2, ANKRD12, MKRN3, RBMX, TCF7L2, ZNF800, MALAT1, SREK1, GKAP1, TRIM59, UHRF1, WNK1, TRPS1, MIB1, STK17B, SCARNA17, TOB1, MDM4, CCDC88A, DCAF8, ZNF638, ANKRD36B, USP47, SYCP3, CDC14A, TRA2B, FAM98B, PPM1K, BDH2, KDM5A, RGPD5, ANKRD10-IT1, SNORD1164, NKTR, FRYL, SPAG9, UBE2D3, SMCHDI, FAM107B, SCFDI, ZBED6, RNPC3, ZFAND6, SMG1, ALS2, PTPRC, PNISR, NUCKS1, TSIX, CNTLN, BRD7, NSUN6, PIGY, CELF2, LUC7L3, DDX59, UBE2Z, PLGLB1, ANKRDI3A, RUFY3, DDX39B, UBE3A, RAB18, LOC100133089, RBM25, CCDC7, BHLHE41, SRRM2, RGPD6, PTEN, AGFG1, RASSF3, AASDH, KDELC2, DACH1, REST, FNIP1, KIF5B, PRKD3, IFT80, C11orf58, PPIG, ZNF138, PARP11, CARD6, MORF4L2, TMTC3, SLC44A1, PYHINI, SNORA32, RRBP1, NEDD1, EPC1, PRPF38B, CI6orf52, MIAT, CCNC, DIS3L2, SEPT7P2, CLTC, RPS16P5, SREKIIPI, PPPIRI2B, NSF, SP100, CAPRIN1, CNTRL, GNAQ, ESF1, TNFAIP8, LOC100129447, FGFR1OP2, EIF3C, SCAMP1, GOLIM4, ZEB2, CADM1, PAIP2B, YLPM1, ZC3H11A, TTN, HBSIL, RHOBTB3, ZNF638IT1, VPS13C, RANBP2, MARVELD2, C3orf38, SCAF11, WHAMMP3, FCHO2 and TOP1

LDHAL6A, FANCC, ARMCX3, SLC26A2, PCDHGB3, TBC1D23, PSMAI, F8, GFM2, DDX6, ZNF326, IL7, FGF5, CDIC SYNE2, PBRM1, RAD51C, LONRF3, RNF13, TIFA and FANCB

SLC25A46, ANKIB1, XIST and ZNF621

$R G S 1$

XAF1, SRGAP1, PATJ, YPEL2, GBP2, LATS2, MRGPRF, ITPRIPL2, GRTP1, MKNK2, ZIC1 and ANGPT2

CXCR4

CD44 and CD163

GFAP

$C 4 B$ and $L T F$

HMGCLL1

$N E L L 1$ 
Table 3 (continued)

Dataset no
GSE122063, GSE15222, GSE48350 and GSE
GSE122063, GSE132903, GSE15222 and
GSE110226, GSE122063 and GSE97760
GSE15222, GSE5281 and GSE97760
GSE122063, GSE5281 and GSE97760
GSE122063, GSE15222 and GSE97760
GSE132903, GSE15222 and GSE5281
GSE122063, GSE15222 and GSE5281
GSE122063, GSE48350 and GSE5281
GSE122063, GSE132903 and GSE5281
GSE122063, GSE132903 and GSE15222
GSE110226 and GSE97760
GSE110226 and GSE5281
GSE110226 and GSE122063
GSE5281 and GSE97760

GSE15222 and GSE97760 GSE122063 and GSE97760

GSE15222 and GSE5281 GSE132903 and GSE5281 GSE122063 and GSE5281

GSE122063 and GSE15222 GSE122063 and GSE48350 GSE122063 and GSE132903 FDR $p$-value $<0.01$ and $\log$ FC $>1$ Upregulated GSE132903, GSE15222, GSE5281 and GSE97760 GSE15222, GSE5281 and GSE97760 GSE122063, GSE5281 and GSE97760 GSE122063, GSE15222 and GSE5281 GSE122063, GSE132903 and GSE5281 GSE122063, GSE15222 and GSE48350 GSE5281 and GSE97760
Common DEGs

$S S T$

RGS4, ENC1, PCSK1, CRYM and NPTX2

$H D C$

$\mathrm{ROBO} 2$

PAX7, TSPAN7, STMN1, WBSCR17, MAP7D2, SULT4A1, INA, NRIP3, DOCK3, IGF1, REEP1, CGREF1, ICA1, SPHKAP, $L A M B 1$ and $\mathrm{ZDHHC} 23$

TAC3

SERPINI

ADCYAPI, ZBBX, NEUROD6, GRP, SLC3OA3, CARTPT, CRH and SERTMI

$A B C C 12, C A L B 1$ and MIR7-3HG

RTN1, PRKCB, NELL2, NEFM, HPRT1, DYNCIII, PARM1, GABRA1, CHGB, GABRG2, RGS7 and SYT1

$V G F$ and $N E C A B 1$

SFRP2, TCF21, ADAMTSL1, EGFEMIP and IGSF1

LYRM9

CTXN3 and NPY2R

ATXN10, DUSP4, SSU72, KIAA1324, SEZ6, SYTL5, DCTN1, TALDO1, FIS1, GPX4, PTP4A3, SNCA, HN1, AP2S1, KCTD2, MCAT, BLVRB, DPP6, NCAM2, ATP6VOC, KCNG3, SYNE1, SPTBN2, ATRNL1, ATP2B3, PTGER3, ATP6VOD1, DNAJA4, LMF1, SGIP1, CROT, ANKS1B, ANK2, SLIT3, SEZ6L2, RNF187, ANKRD54, CALY, TSPAN5, CSRNP3, MFSD2B, HGD, DAB2IP, CX3CLI, RANBP10, AHNAK2, DPCD, PAK1, NOC4L, UBL7, HAGH, ASPSCR1, TRAPPC5, CNKSR2, LOC729870, DCAF6, CD99L2, PTPRF, CPNE6, RNF24, TBCID7, NAV3, ATP6VIG2, TMEM59L, SLC24A3, MLXIP, TSTA3, FOLH1, SPTAN1, TCEA2, AP2M1, SMOX, FHL2, ASCC2, PRDX5, FKBP1B, HYDIN, AP3B2, PDE1A, FAM13IA, TMEM158, NFIB, UMODL1, MEG3 and GCAT

$D G K B$ and CORT

GLT1D1, NOS2, XK, FAM182B, PTPN5, RTN4RL1, NECAB2, PRRT1, LOC284395, SSX3, KIAA1045, NKX2-3, PVALB, CHRFAM7A, KIAA1239, GSG1, ADCY2, FAM178B, GLP2R, LOC100289580, WNK2, GYG2P1, LRRC38, DDAH1, TBXA2R, RET, LOC100507534, ZSCAN1, OCA2, HAPLNI, INSL3, ENTPD3, KATNB1, RPL13AP17, NAALADL2, ST7-AS1, NPPA, SLC7A4, PCDHIIX, RPH3A, CASOI, ODZ3, NGEF, KIAA1644, LOC653550, MYO5B, PNMA5, LOC338797, KCNH2, TUBA3C, LOC100288814, LOC497256, DRGX, GPR88, CHRM2, PRKARIB, FLJ32255, LOC100134259, SLC22A10 and PVRL3-ASI

GABRA5, ANO3, APIS1, SERINC3, ITFG1, ICAM5, PGM2L1, CCK, PLK2 and NCALD

GLRB, ERICH3, TUBB2A and NSF

GDA, MET, SERPINF1, LINC00460, ZNF385B, SYT13, LOC283484, SARS, CHRM1, CHRNB2, GPATCH2, KRT222, NMNAT2, UBE2N, ZCCHC12, GPR158, SDR16C5, FGF12, FPGT-TNNI3K, TAC1, RNF175, UBE2QL1, SYN2, ATL1, AMPH, MYTIL, NAPIL5, TAGLN3, Cl4orf79, UNCl3A, SOSTDC1, SH3GL2, STMN2, MAP4, MDHI, STAT4, VSNLI, GPRASP2, EPHA5, TRIM37, FAR2, PCLO, SV2B, SVOP, PAK3, CDC42, CAMKIG, PPPIR2, NOP56, PTPRO, BSCL2, CIRBP, HS6ST3, PPPIR14C, SCG5, NPTXR, GLS2, GOLT1A, TASP1, ACOT7, RSPO2, ENO2, NEFL, CD200, RBM3, GAP43, ERC2, GNG2, PPMIE, RPS4Y1, TARBP1, SLC1A6, GNG3, NECAP1, GABRD, GLS, LINCO0467, NRXN3, LY86-AS1, ATP8A2, MLLT11, BRWDI, PPMIJ, RAB3C, UCHL1, WDR54, BDNF, DCLK1, PNMAL2, CITED1, NUDT18, RAB27B, SNAP25, GOLGA8A, HMP19, LOC100506124, SYCE1, CCKBR, TUBB3, COPG2IT1, RBP4, PPEF1, CACNG3, MICAL2, LOC100129973, PTPN3, PLD3, ATOH7, MAL2 and BEX5

SCG2, VIP, KCNVI, TMEM155, NMU, HSPB3 and PCDH8

SLC 32AI

CAP2

SLC5A3 and SERPINA3

RHOQ and IL6ST

FAM107B, ZBED6, NEATI, RRBPI and TTN

$G B P 2$ and $A N G P T 2$

GFAP

$C 4 B$ and $L T F$

USP47, CHD9, IPW, TRA2B, FAM98B, PPMIK, BDH2, KDM5A, OKI, RGPD5, ANKRDIO-ITI, IL6R, SNORDI16-4, NKTR, FRYL, PTPN2, AHNAK, UBE2W, JPX, RDX, FAM161A, ZMYM5, SET, FAM120A, SNORA18, BDP1, C5orf56, UBE2D3, YTHDC2, SMCHD1, CCDC144A, TAFID, ZNF713, SNRNP48, SNORD107, RNPC3, SNORD50B, LRRFIP1, ELK4, ALS2, PTPRC, GRAMDIC, PNISR, SNORD61, LMO7, NUCKS1, CNTLN, SAMHD1, PTBP3, TRIM4, CXCL2, TNPO1, CDK13, ZFP36L1, STAG1, BRD7, SKI, TBLIXR1, SNORA1, ANKRD36, CPEB4, NSUN6, MKL2, PIGY, HCG18, ZNF160, CELF2, LUC7L3, MECOM, DDX59, UBE2Z, ZDHHC21, CBX3, ANKRD13A, TFEB, RUFY3, SKIL, UBE3A, TLE4, RAB18, LOC100133089, RBM25, KCNJ16, CCDC7, KTN1, RGPD6, SAT1, ABLIM1, ZNF280D, RBMS1, LPP, ATRX, MACF1, PCMTD2, AGFG1, RASSF3, AASDH, C5orf24, KDELC2, SFPQ, ZSCAN30, STAG2, RBM33, RAPH1, REST, FNIP1, KIF5B, SNORA40, PPIG, ZNF138, ZNF566, PIK3C2A, PARP11, NOTCH2NL, LEF1, MORF4L2, TMTC3, NEK1, SLC44A1, PYHIN1, SNORD5, NEDDI, EPC1, PRPF38B, Cl6orf52, MIAT, SEPT7, CCNC, DIS3L2, SEPT7P2, PTAR1, TUBE1, SREKIIPI, NSF, USP6, SPIOO, CAPRINI, ZBTBI, CNTRL, SNORA8, TP53INPI, GNAO, ESFI, TNFAIP8, SNORD84, FGFR1OP2, EIF3C, FAM185A, SCAMP1, GOLIM4, ZEB2, CADM1, ANKRD12, YLPM1, ZC3H11A, RBMX, HBSIL, ZNF800, RHOBTB3, MALAT1, SREK1, GKAP1, UHRF1, WNK1, VPS13C, TRPS1, RANBP2, C3orf38, SCAF11, VSIG10, WHAMMP3, FCHO2, MIB1, STK17B, SCARNA17, TOB1, MDM4, CCDC88A and DCAF8

SLC26A2, FGF5, TBC1D23, PSMA1, PBRM1, RAD51C, F8, LONRF3, DDX6, ZNF326 and FANCB

SLC25A46, XIST, ZNF621 and ANKIB1 
Table 3 (continued)

\begin{tabular}{|c|c|}
\hline Dataset no & Common DEGs \\
\hline GSE122063 and GSE97760 & $\begin{array}{l}\text { AHSA2, CHORDC1, EIF4G3, CCDC66, LOC100287765, Q5A5F0, SNORA75, MSR1, F13A1, WDR33, LOC100507645, } \\
\text { ZNF620, IL18R1, SERPINA3, ZNF850, AFF1, GON4L, RUNX1, IL1RL1, LOC387895, CA5BP1, SNORA73A, CXCL12, } \\
\text { RBM47, LRRC37A3, EFTUD1, LOC100129089, SPATA13 and PLAC8 }\end{array}$ \\
\hline GSE15222 and GSE5281 & MRGPRF, ITPRIPL2, XAF1, GRTP1, MKNK2, SRGAP1, PATJ, YPEL2, ZIC1 and LATS2 \\
\hline GSE48350 and GSE5281 & CXCR4 \\
\hline GSE122063 and GSE5281 & $\begin{array}{l}\text { CD44, HIGD1B, BACE2, PIEZO2, SOCS3, CEP104, EGFR, PDLIM4, ITPKB, RHOJ, PDE4DIP, VASP, COL27A1, MAFF, } \\
\text { KCNE4, SCIN, MYO10, SNX31, ZFP36L2, EMP1, SLCOIA2, TNS1, SRGN, SLCO4A1, CD163, TBL1X, CXCL1, BCAS1, } \\
\text { TNFRSF10B, FAM65C and LOC100131541 }\end{array}$ \\
\hline GSE122063 and GSE15222 & FOXJ1, MIA, S100A12, S100A4 and C21orf62 \\
\hline GSE122063 and GSE48350 & $C 4 A$ \\
\hline \multicolumn{2}{|l|}{ Downregulated } \\
\hline GSE122063, GSE15222, GSE48350 and GSE5281 & SST and BDNF \\
\hline GSE122063, GSE132903, GSE15222 and GSE5281 & RGS4, CRYM, NPTX2, RTN3 and ENCl \\
\hline GSE15222, GSE5281 and GSE97760 & ROBO2 \\
\hline GSE122063, GSE5281 and GSE97760 & $\begin{array}{l}\text { IGF1, STMN1, REEP1, CGREF1, ICA1, SPHKAP, WBSCRI7, MAP7D2, SULT4A1, LAMB1, ZDHHC23, NRIP3, HMGCLL1 } \\
\text { and DOCK3 }\end{array}$ \\
\hline GSE122063, GSE15222 and GSE97760 & TAC3 \\
\hline GSE122063, GSE15222 and GSE5281 & ADCYAP1, CRH, ZBBX, NEUROD6, SLC3OA3, NELL1, CARTPT and SERTM1 \\
\hline GSE122063, GSE48350 and GSE5281 & $A B C C 12, C A L B 1$ and $M I R 7-3 H G$ \\
\hline GSE122063, GSE132903 and GSE5281 & RTN1, PRKCB, NELL2, GABRA1, CHGB, GABRG2, NEFM, RGS7, SYT1, HPRT1, DYNC1II and PARM1 \\
\hline GSE122063, GSE132903 and GSE15222 & PCSKI, VGF and NECABI \\
\hline GSE5281 and GSE97760 & $\begin{array}{l}\text { NOC4L, ATXN10, DUSP4, SSU72, KIAA1324, SEZ6, UBL7, DCTN1, HAGH, ASPSCR1, FIS1, PTP4A3, SNCA, HN1, AP2S1, } \\
\text { KCTD2, MCAT, CNKSR2, BLVRB, DCAF6, CD99L2, ATP6VOC, CPNE6, SYNE1, TBC1D7, NAV3, ATP6V1G2, TMEM59L, } \\
\text { ATRNL1, MLXIP, LMF1, SPTAN1, SGIP1, CROT, SMOX, FHL2, ASCC2, SEZ6L2, CALY, FKBP1B, TSPAN5, FAM131A, } \\
\text { TMEM158, DAB2IP, CX3CL1, MEG3, GCAT and DPCD }\end{array}$ \\
\hline GSE15222 and GSE97760 & CORT and $D G K B$ \\
\hline GSE122063 and GSE97760 & $\begin{array}{l}\text { XK, KATNB1, FAM182B, RPL13AP17, PTPN5, RTN4RL1, ST7-AS1, NPPA, PRRT1, PCDH11X, LOC284395, SSX3, } \\
\text { KIAA1045, CASQ1, ODZ3, KIAA1644, NKX2-3, PVALB, CHRFAM7A, KIAA1239, GSG1, ADCY2, FAM178B, } \\
\text { LOC100289580, WNK2, MYO5B, PNMA5, LOC338797, KCNH2, RET, LOC497256, LOC100507534, ZSCAN1, GPR88, } \\
\text { CHRM2, PRKAR1B, FLJ32255, SLC22A10, PVRL3-AS1 and OCA2 }\end{array}$ \\
\hline GSE15222 and GSE5281 & PGM2L1, GABRA5, ANO3, AP1S1, SERINC3, CCK, PLK2, NCALD and ICAM5 \\
\hline GSE132903 and GSE5281 & ERICH3, TUBB2A, NSF and GLRB \\
\hline GSE122063 and GSE5281 & $\begin{array}{l}\text { PAX7, GDA, MET, SERPINF1, LINCO0460, SYT13, LOC283484, TASP1, TSPAN7, ACOT7, SARS, CHRM1, CHRNB2, } \\
\text { GPATCH2, KRT222, NMNAT2, UBE2N, ZCCHC12, GPR158, SDR16C5, ENO2, FGF12, CD200, FPGT-TNNI3K, RBM3, } \\
\text { GAP43, ERC2, GNG2, RNF175, PPM1E, TARBP1, UBE2QL1, SYN2, ATL1, AMPH, SLC1A6, GNG3, NECAP1, MYTIL, } \\
\text { NAPIL5, TAGLN3, C14Orf79, GABRD, UNC13A, GLS, SOSTDC1, NRXN3, LY86-AS1, ATP8A2, SH3GL2, MLLT11, STMN2, } \\
\text { BRWD1, MAP4, PPMIJ, RAB3C, UCHL1, WDR54, MDH1, BDNF, DCLK1, STAT4, VSNL1, GPRASP2, EPHA5, PNMAL2, } \\
\text { CITED1, NUDT18, TRIM37, FAR2, PCLO, SV2B, RAB27B, SNAP25, GOLGA8A, HMP19, SVOP, LOC100506124, PAK3, } \\
\text { CDC42, SYCE1, CAMK1G, CCKBR, TUBB3, COPG2IT1, PPPIR2, RBP4, PPEF1, NOP56, INA, CACNG3, MICAL2, } \\
\text { PTPRO, LOC100129973, BSCL2, PTPN3, CIRBP, PLD3, HS6ST3, PPPIR14C, ATOH7, SCG5, MAL2, NPTXR, BEX5 and } \\
\text { GLS2 }\end{array}$ \\
\hline GSE132903 and GSE15222 & SERPINII \\
\hline GSE132903 and GSE15222 & SCG2, VIP, KCNV1, GRP, NMU, HSPB3, TMEM155 and PCDH8 \\
\hline GSE122063 and GSE48350 & $S L C 32 A 1$ \\
\hline GSE122063 and GSE132903 & $C A P 2$ \\
\hline
\end{tabular}

the number of datasets was not equal, and the common DEGs were not seen in $60 \%$ of the datasets. Therefore, this criterion was rejected.

\section{(iv) FDR $p$-value $<0.01$ and $\log$ FC $>1$}

Among the 16 datasets, this criterion was met by six datasets containing both upregulated and downregulated DEGs (Tables 2 and 3). Common upregulated and downregulated DEGs were found in four datasets which accounted for more than $60 \%$. Hence, this criterion was selected to retrieve the DEGs for PPI and functional enrichment analysis. Among upregulated DEGs, solute carrier family 5 member 3 (SLC5A3) and serpin family A member 3 (SERPINA3) were found to be common in four datasets. Among downregulated DEGs, somatostatin (SST), regulator of G protein signaling 4 (RGS4), crystallin mu (CRYM), neuronal pentraxin 2 (NPTX2), reticulon 3 (RTN3), brain-derived neurotrophic factor $(B D N F)$ and ectodermal-neural cortex 1 (ENCl) genes were found to be common in four datasets (Fig. 4). These genes were selected for further PPI analysis with LDGs.

\section{PPI Analysis}

Eighteen LDGs were selected from the NCBI portal (Table 4) and were subjected to PPI analysis with the 
a

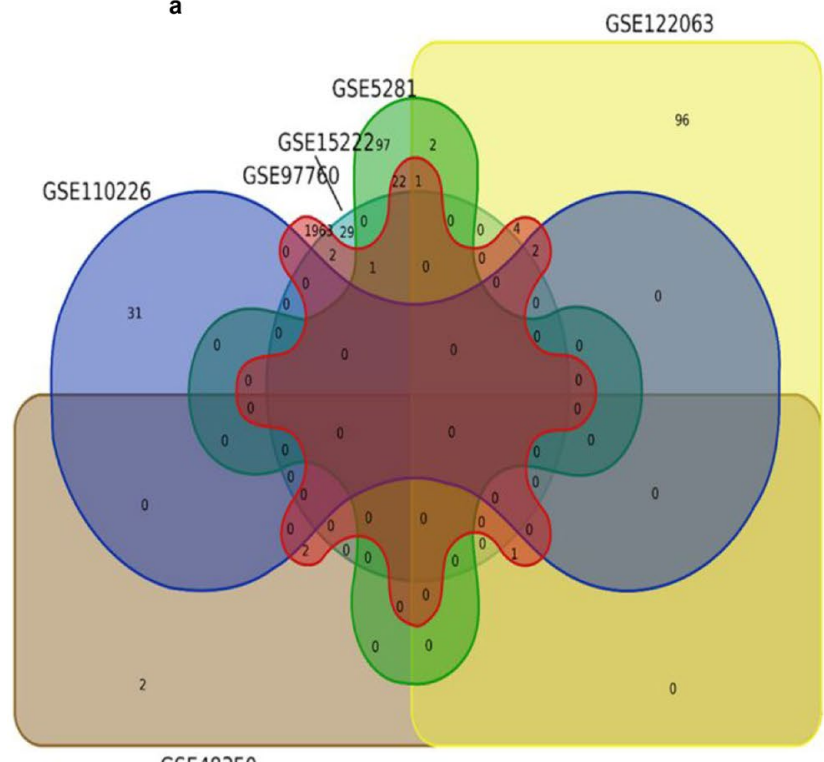

b

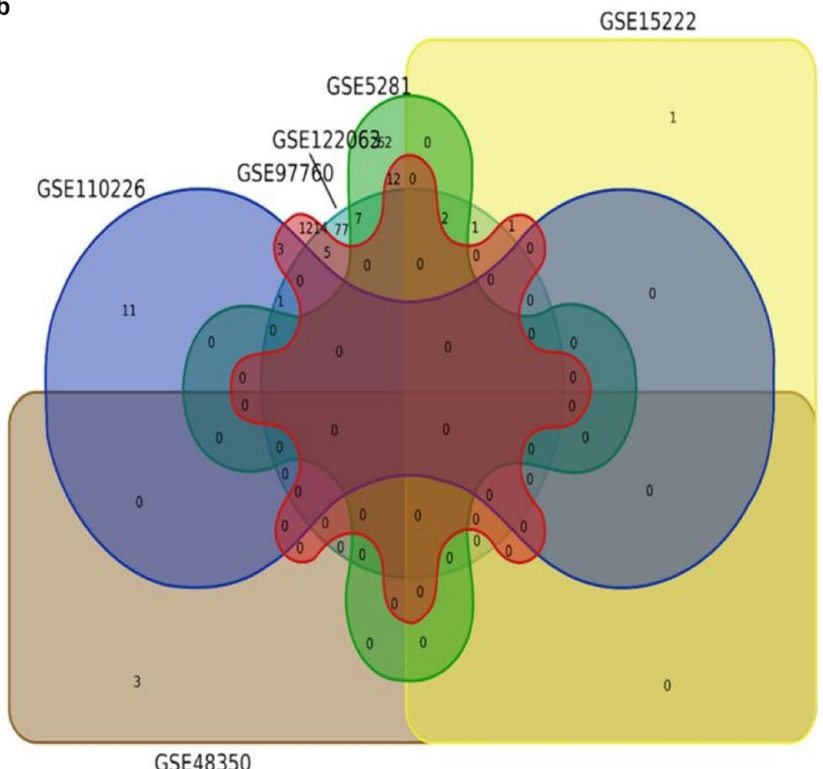

Fig. 3 Venn diagram exhibiting the common upregulated (a) and downregulated (b) DEGs

shortlisted DEGs from the above step. PPI analysis (Fig. 5) revealed that BDNF exhibited the highest node degree (16), followed by SST (7), AACT (SERPINA3) (4), RTN3 (2), RGS4 (3), NPTX (1) and CRYM (1). BDNF exhibited high connectivity with AD-specific proteins including glutamate ionotropic receptor NMDA type subunit 2B (GRIN2B), BACE1, MAPT, PSEN1, TP53, BCHE, SNCA, COMT, INS, APP, APOE and ACHE. SST exhibited PPI with IDE, MME, IGF, APP, INS and ACHE. SERPINA3/AACT exhibited interactions with APOA1, APOE and APP proteins.
RTN3 interacted with BACE1 and APP. RGS4 interacted with COMT alone. NPTX and CRYM did not exhibit interactions with any of the LDGs (Fig. 5, Tables 5 and 6).

\section{Functional Enrichment Analysis}

The common DEGs retrieved were subjected to functional enrichment analysis to explore their involvement in Gene Ontology (GO) and Kyoto Encyclopedia of Genes and Genomes (KEGG) pathways.
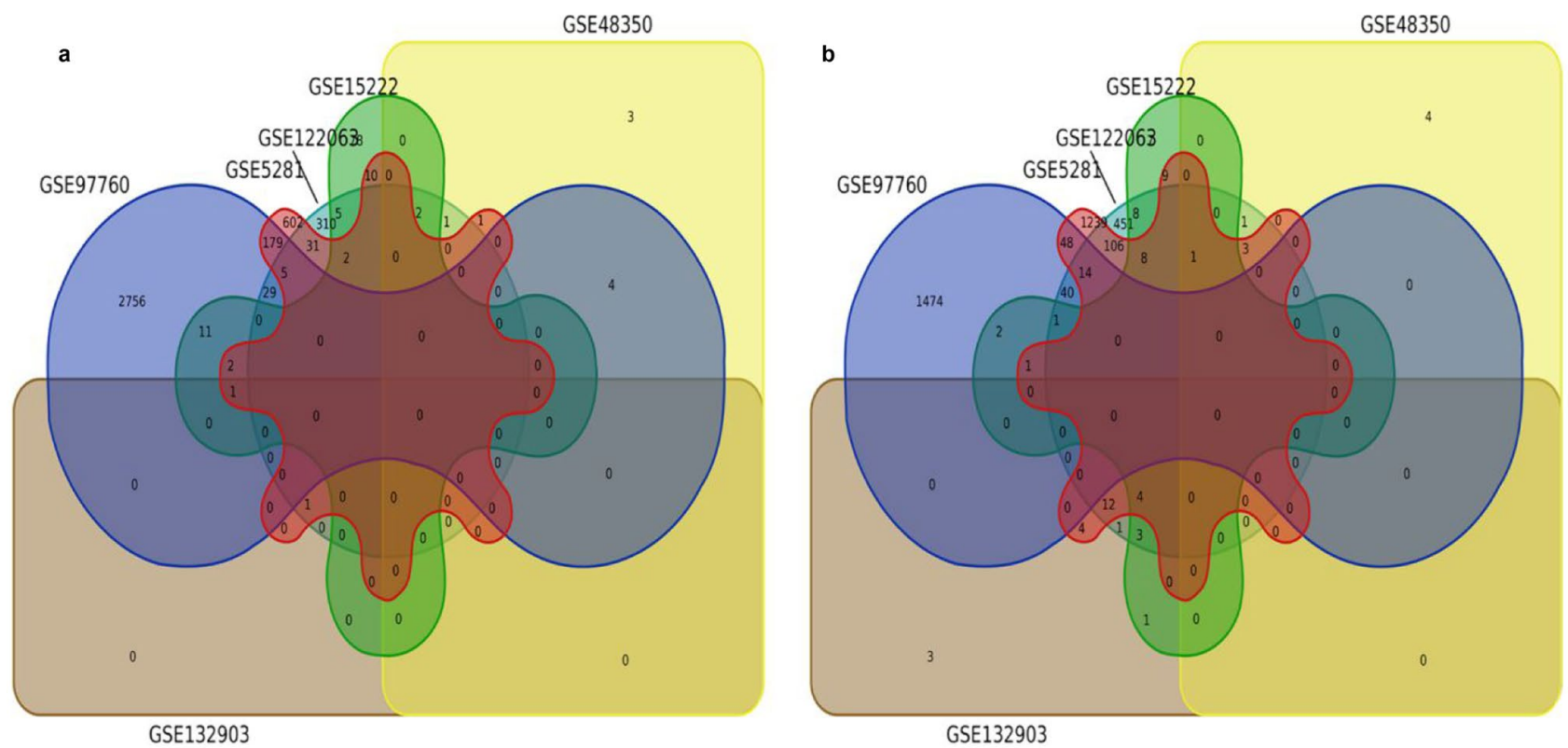

Fig. 4 Venn diagram exhibiting the common upregulated (a) and downregulated (b) DEGs 
Table 4 List of LDGs retrieved from NCBI

\begin{tabular}{|c|c|c|c|c|}
\hline Gene symbol & NCBI gene ID & $\begin{array}{l}\text { HUGO Gene Nomenclature Com- } \\
\text { mittee (HGCN) ID }\end{array}$ & $\begin{array}{l}\text { Chromosome } \\
\text { location }\end{array}$ & Reference \\
\hline APOE & 348 & HGNC:613 & $19 \mathrm{q} 13.32$ & (Nho et al. 2017) \\
\hline$A P P$ & 351 & HGNC:620 & $21 \mathrm{q} 21.3$ & (Schrötter et al. 2012) \\
\hline GRIN2B & 2904 & HGNC:4586 & $12 \mathrm{p} 13.1$ & (Andreoli et al. 2013) \\
\hline$S N C A$ & 6622 & HGNC: 11,138 & $4 \mathrm{q} 22.1$ & (Mackin et al. 2015) \\
\hline$M A P T$ & 4137 & HGNC:6893 & $17 \mathrm{q} 21.31$ & (Sassi et al. 2014) \\
\hline COMT & 1312 & HGNC:2228 & $22 \mathrm{q} 11.21$ & (Zhou et al. 2013) \\
\hline TP53 & 7157 & HGNC:11,998 & $17 \mathrm{p} 13.1$ & (Wojsiat et al. 2017) \\
\hline$A G E R$ & 177 & HGNC:320 & $6 \mathrm{p} 21.32$ & (Deane et al. 2003) \\
\hline$I G F 1$ & 3479 & HGNC:5464 & $12 \mathrm{q} 23.2$ & (Majores et al. 2002) \\
\hline PSEN1 & 5663 & HGNC:9508 & $14 \mathrm{q} 24.2$ & (Sassi et al. 2014) \\
\hline$B A C E 1$ & 23,621 & HGNC:933 & $11 \mathrm{q} 23.3$ & (Kimura et al. 2016) \\
\hline INS & 3630 & HGNC:6081 & $11 \mathrm{p} 15.5$ & (Majores et al. 2002) \\
\hline$A P O A 1$ & 335 & HGNC:600 & $11 \mathrm{q} 23.3$ & (Fitz et al. 2015) \\
\hline$L D L R$ & 3949 & HGNC:6547 & $19 \mathrm{p} 13.2$ & (Shinohara et al. 2017) \\
\hline ACHE & 43 & HGNC:108 & $7 \mathrm{q} 22.1$ & (Scacchi et al. 2009) \\
\hline BCHE & 590 & HGNC:983 & $3 \mathrm{q} 26.1$ & (Scacchi et al. 2009) \\
\hline$I D E$ & 3416 & HGNC:5381 & $10 \mathrm{q} 23.33$ & (Jha et al. 2015) \\
\hline$N E P$ & 4311 & HGNC:7154 & $3 q 25.2$ & (Jha et al. 2015) \\
\hline
\end{tabular}

Fig. 5 PPI network of DEGs exhibiting significant interactions with LDGs. Yellow nodes represent common genes retrieved from GEO datasets. Pink nodes represent LDGs

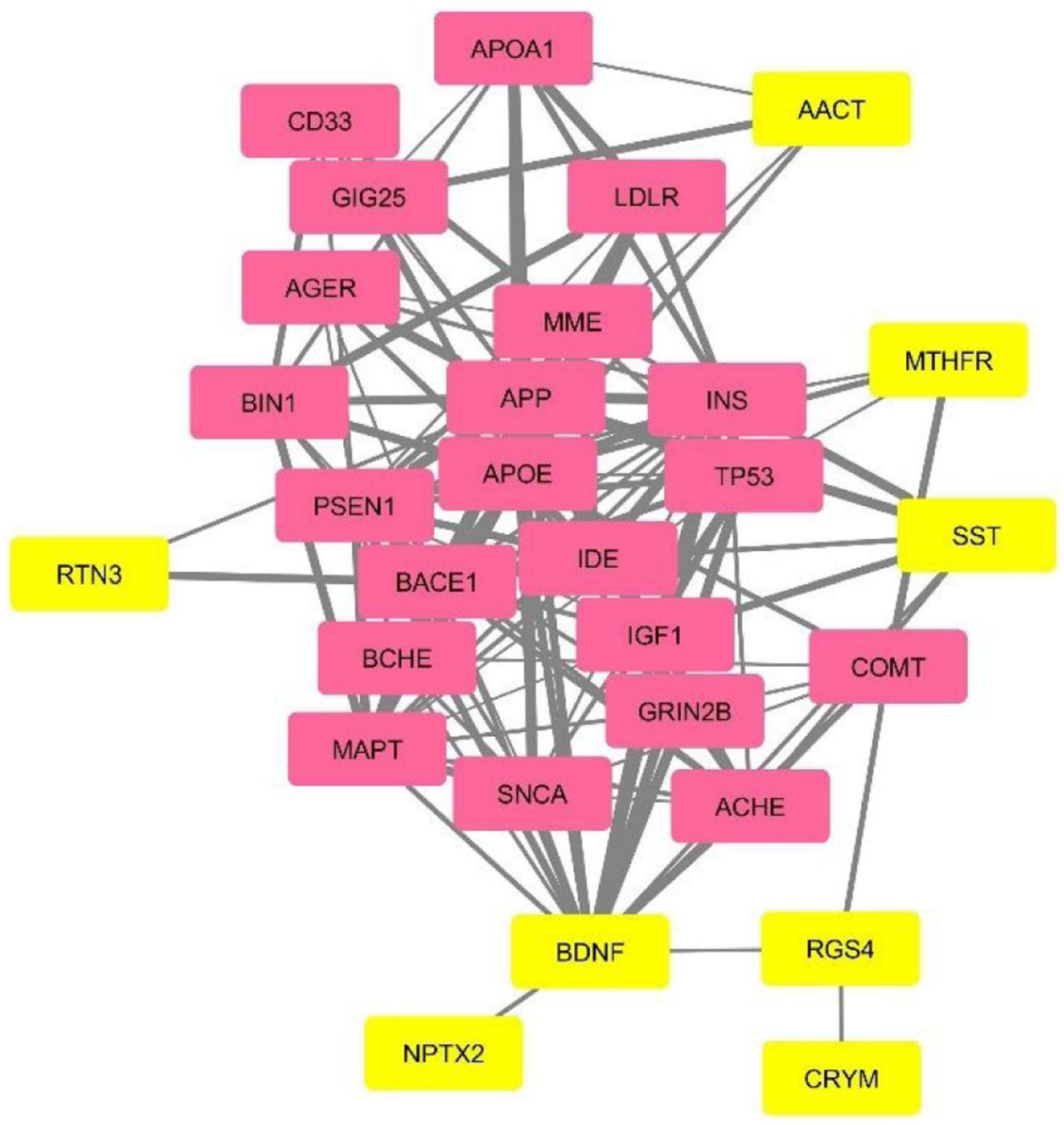


Table 5 Significant PPI of identified DEGs with LDGs

\begin{tabular}{|c|c|c|}
\hline Node 1 & Node 2 & $\begin{array}{l}\text { Combined } \\
\text { score* }\end{array}$ \\
\hline \multirow[t]{13}{*}{ BDNF } & TP53 & 0.95 \\
\hline & IGF1 & 0.894 \\
\hline & APP & 0.828 \\
\hline & APOE & 0.81 \\
\hline & PSEN1 & 0.781 \\
\hline & COMT & 0.733 \\
\hline & INS & 0.715 \\
\hline & SNCA & 0.708 \\
\hline & ACHE & 0.657 \\
\hline & MAPT & 0.598 \\
\hline & BACE1 & 0.594 \\
\hline & BCHE & 0.518 \\
\hline & GRIN2B & 0.982 \\
\hline \multirow[t]{6}{*}{ SST } & APP & 0.928 \\
\hline & INS & 0.915 \\
\hline & IGF1 & 0.791 \\
\hline & IDE & 0.59 \\
\hline & ACHE & 0.579 \\
\hline & $\mathrm{MME} / \mathrm{NEP}$ & 0.404 \\
\hline \multirow[t]{3}{*}{ AACT/SERPINA3 } & APP & 0.476 \\
\hline & APOA1 & 0.45 \\
\hline & APOE & 0.609 \\
\hline \multirow[t]{2}{*}{ RTN3 } & APP & 0.523 \\
\hline & BACE1 & 0.8 \\
\hline RGS4 & COMT & 0.641 \\
\hline
\end{tabular}

*Combined score-Computed based on the evidence gathered from sources such as literature-derived co-expression and co-occurrences, database imports, gene fusions, large-scale experimental reports, and phylogenetic co-occurrences. Combined score $<0.4$ is considered as low confidence; $0.4-0.7$ as medium confidence; and above 0.7 is acknowledged as high confidence

GO analysis revealed that $S L C 5 A 3$ was involved in the transport of potassium ions across plasma membranes (GO:0098739) and peripheral nervous system development (GO:0007422), whereas BDNF, RGS4, NPTX2 and SST were involved in cognitive ability (GO:0050890), transsynaptic signaling (GO:0099157), striated muscle cell differentiation (GO:0051154), anterograde trans-synaptic transmission (GO:0098916) and regulation of nervous system processes (GO:0031644). BDNF, SST and ENCl were involved in receptor ligand activity (GO:0048018), cytokine receptor binding (GO:0005126), positive regulation of cell projection organization (GO:0031346) and receptor regulator activity (GO:0030545). ENCl and RTN3 were found to be involved in negative regulation of cellular amide metabolic process (GO:0034249). SERPINA3 in combination with $S S T$ was known to be involved in digestion (GO:0007586) (Fig. 6).

KEGG analysis revealed that $B D N F$ was involved in triggering the phosphoinositide 3-kinase (PI3K) pathway (hsa04213), rat sarcoma (RAS) signaling (hsa05212), RAC1 signaling (hsa04510), FYN signaling (hsa04380), cyclin-dependent kinase 5 (CDK5) phosphorylation,
FYN-mediated GRIN2B activation and transcriptional signaling. $B D N F$ and $S S T$ were involved in transcription regulation by methyl-CpG-binding protein 2 (MECP2), gastric acid secretion (hsa04971) and somatostatin gene expression. RGS4 was known to mediate G alpha (i) auto-inactivation and $\mathrm{G}$ alpha (q) inactivation by hydrolysis of guanosine triphosphate (GTP) to guanosine diphosphate (GDP). CRYM was involved in lysine catabolism and autosomal-dominant deafness, whereas $R T N 3$ was involved in PPI at synapses, binding of synaptic adhesion-like molecule 1-4 (SALM1-4) to reticulons and synaptic adhesion-like molecules. SERPINA3 was involved in exocytosis of platelet alpha granules and azurophil granule lumen proteins (Fig. 7).

\section{Discussion}

This study was aimed to retrieve significant DEGs associated with $\mathrm{AD}$ by analyzing the gene expression data available in the GEO database. Initially, the GEO datasets were selected based on the inclusion and exclusion criteria, which resulted in 32 datasets. The raw data for each dataset were analyzed individually using the Bioconductor package in $\mathrm{R}$, and DEGs with FDR $p$-value $<0.05$ were retrieved and segregated into upregulated and downregulated DEGs. Although 32 datasets were found to be eligible, only 16 satisfied the initial criteria FDR $p$-value $<0.05$. These DEGs were subjected to screening based on different filtering norms, and this yielded six datasets with both upregulated and downregulated DEGs. Herein, the overlapping DEGs were found in more than $60 \%$ of the above mentioned six datasets. SLC5A3 and SERPINA3 were found to be common in upregulated DEGs, whereas SST, BDNF, RGS4, CRYM, $N P T X 2, R T N 3$ and $E N C 1$ were found to be common in downregulated DEGs. These DEGs were further subjected to PPI analysis with 18 LDGs which were known to play a strong role in $\mathrm{AD}$ pathogenesis. Among the above nine DEGs, BDNF, SST, SERPINA3 (AACT), RTN3 and RGS4 exhibited significant interactions.

BDNF exhibited interaction with crucial targets including GRIN2B, BACE1, APP, MAPT, SNCA, ACHE, APOE, PSEN1 and COMT. Functional enrichment analysis revealed a normal physiological role of BDNF in cytokine signaling, receptor ligand activity and regulation, trans-synaptic signaling, cognitive function, chemical synaptic transmission, cell differentiation, cell growth and regulation. This suggests its crucial involvement in neuronal growth, development and transmission, which is found to be abnormal in AD. KEGG pathway analysis revealed detailed mechanistic action of BDNF. BDNF initiates its response by binding to the tyrosine kinase beta (TRK $\beta$ ) receptor; post-binding, the receptor dimerizes and undergoes autophosphorylation. The phosphorylated TRK $\beta$ triggers various signaling mechanisms 
Table 6 Characteristics of the PPI network

\begin{tabular}{|c|c|c|c|c|c|c|c|}
\hline Node name & $\begin{array}{l}\text { Average shortest path } \\
\text { length }^{\text {a }}\end{array}$ & $\begin{array}{l}\text { Betweenness cen- } \\
\text { trality }^{\mathbf{b}}\end{array}$ & Clustering coefficient ${ }^{\mathrm{c}}$ & $\begin{array}{l}\text { Node } \\
\text { degree }^{d}\end{array}$ & $\begin{array}{l}\text { Neighborhood con- } \\
\text { nectivity }^{\mathrm{e}}\end{array}$ & Radiality $^{f}$ & $\begin{array}{l}\text { Topological coef- } \\
\text { ficient }^{\mathrm{g}}\end{array}$ \\
\hline APP & 1.214286 & 0.167659 & 0.399209 & 23 & 10.26087 & 0.946429 & 0.380032 \\
\hline APOE & 1.214286 & 0.171997 & 0.403162 & 23 & 10.3913 & 0.946429 & 0.384863 \\
\hline PSEN1 & 1.392857 & 0.045109 & 0.555556 & 18 & 12.05556 & 0.901786 & 0.446502 \\
\hline INS & 1.392857 & 0.055697 & 0.542484 & 18 & 12 & 0.901786 & 0.444444 \\
\hline BACE1 & 1.428571 & 0.052044 & 0.573529 & 17 & 12.29412 & 0.892857 & 0.455338 \\
\hline BDNF & 1.428571 & 0.1859 & 0.525 & 16 & 11.875 & 0.892857 & 0.4375 \\
\hline MAPT & 1.535714 & 0.014431 & 0.703297 & 14 & 13.71429 & 0.866071 & 0.507937 \\
\hline SNCA & 1.642857 & 0.00439 & 0.836364 & 11 & 15.27273 & 0.839286 & 0.565657 \\
\hline TP53 & 1.642857 & 0.011054 & 0.763636 & 11 & 14.90909 & 0.839286 & 0.552189 \\
\hline ACHE & 1.642857 & 0.009583 & 0.745455 & 11 & 15.09091 & 0.839286 & 0.558923 \\
\hline IGF1 & 1.678571 & 0.003503 & 0.844444 & 10 & 15.8 & 0.830357 & 0.585185 \\
\hline BCHE & 1.714286 & 0.002774 & 0.861111 & 9 & 16.44444 & 0.821429 & 0.609054 \\
\hline IDE & 1.75 & 0.004939 & 0.781818 & 11 & 14.36364 & 0.8125 & 0.574545 \\
\hline COMT & 1.75 & 0.035647 & 0.464286 & 8 & 10.375 & 0.8125 & 0.384259 \\
\hline SST & 1.785714 & 0.003489 & 0.761905 & 7 & 14.14286 & 0.803571 & 0.52381 \\
\hline MME & 1.785714 & 0.007593 & 0.711111 & 10 & 14 & 0.803571 & 0.56 \\
\hline GRIN2B & 1.821429 & 0.001563 & 0.8 & 6 & 17 & 0.794643 & 0.62963 \\
\hline LDLR & 1.892857 & 0.001436 & 0.857143 & 7 & 16.28571 & 0.776786 & 0.651429 \\
\hline APOA1 & 1.892857 & 0.005669 & 0.666667 & 7 & 12.42857 & 0.776786 & 0.497143 \\
\hline AGER & 1.892857 & 0 & 1 & 7 & 17.14286 & 0.776786 & 0.685714 \\
\hline AACT & 2 & 0 & 1 & 4 & 14.25 & 0.75 & 0.57 \\
\hline GIG25 & 2 & 0 & 1 & 4 & 14.25 & 0.75 & 0.57 \\
\hline RTN3 & 2.142857 & 0 & 1 & 2 & 20 & 0.714286 & 0.869565 \\
\hline RGS4 & 2.25 & 0.071429 & 0.333333 & 3 & 8.333333 & 0.6875 & 0.470588 \\
\hline NPTX2 & 2.392857 & 0 & 0 & 1 & 16 & 0.651786 & 0 \\
\hline CRYM & 3.214286 & 0 & 0 & 1 & 3 & 0.446429 & 0 \\
\hline
\end{tabular}

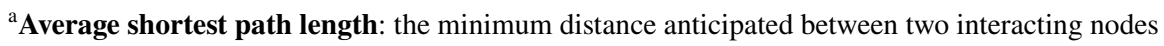

${ }^{b}$ Betweenness centrality: network analysis parameter which indicates the degree of influence of a specific node over other node's interactions

${ }^{c}$ Clustering coefficient: the number of nodal triads that pass through a single node in comparison with maximum number of nodal triads that a node could possess

${ }^{\mathrm{d}}$ Node degree: the number of interactions exhibited by a specific node with other nodes (represented in Cytoscape)

e Neighborhood connectivity: the average connectivity of a particular node with all its neighboring nodes

${ }^{\mathrm{f}}$ Radiality: shortest distance between interacting nodes

${ }^{g}$ Topological coefficient: calculated for those nodes showcasing multiple nodal interactions. It represents the extent of a specific node to share its neighbor with other nodes

such as PI3K, RAS, CDK5, RAC1 GTPase, Src homology 2 domain-containing 1 (SHC1), FYN kinase, fibroblast growth factor receptor substrate 2 (FRS2), T-lymphoma invasion and metastasis-inducing protein 1 (TIAM1) and phospholipase $\mathrm{C}$ gamma 1 (PLCG1). These were in turn found to be involved in triggering secondary signaling pathways through GRIN2B, which is associated with cocaine addiction, cognitive central hypoventilation syndrome and eating disorders. A number of research studies have reported downregulation of $B D N F$ expression, which is in line with our findings (Kang et al. 2020; Akhtar et al. 2020).

The PPI analysis of SST revealed its interaction with primary AD targets including IDE, MME, IGF, APP, INS and ACHE. Like BDNF, SST also exhibited a physiological role in trans-synaptic signaling, cognitive function, anterograde trans-synaptic signaling, receptor ligand activity, cytokine receptor binding and receptor regulator activity. KEGG pathway analysis revealed the association of SST with MECP2 and c-AMP responsive element-binding protein 1 (CREB1). It is reported that MECP2 together with CREB1 enhances the expression of SST by binding to the promoter region (Chahrour et al. 2008). There are five subtypes of SST receptors, of which three receptors, i.e., SSTR2, SSTR4 and SSTR5, were observed to display marked downregulation and reduced sensitivity in AD. This interferes with their inhibitory control over the adenylyl cyclase (AC) pathway. Decreased SSTR2 results in decreased activity of neprilysin, an enzyme involved in the degradation of $\mathrm{A} \beta$ peptides (Burgos-Ramos et al. 2008; Aguado-Llera et al. 2018; Sandoval et al. 2019). In addition, postmortem AD brains with decreased levels of SST receptors were correlated with a higher degree of amnesia and cognitive dysfunction 


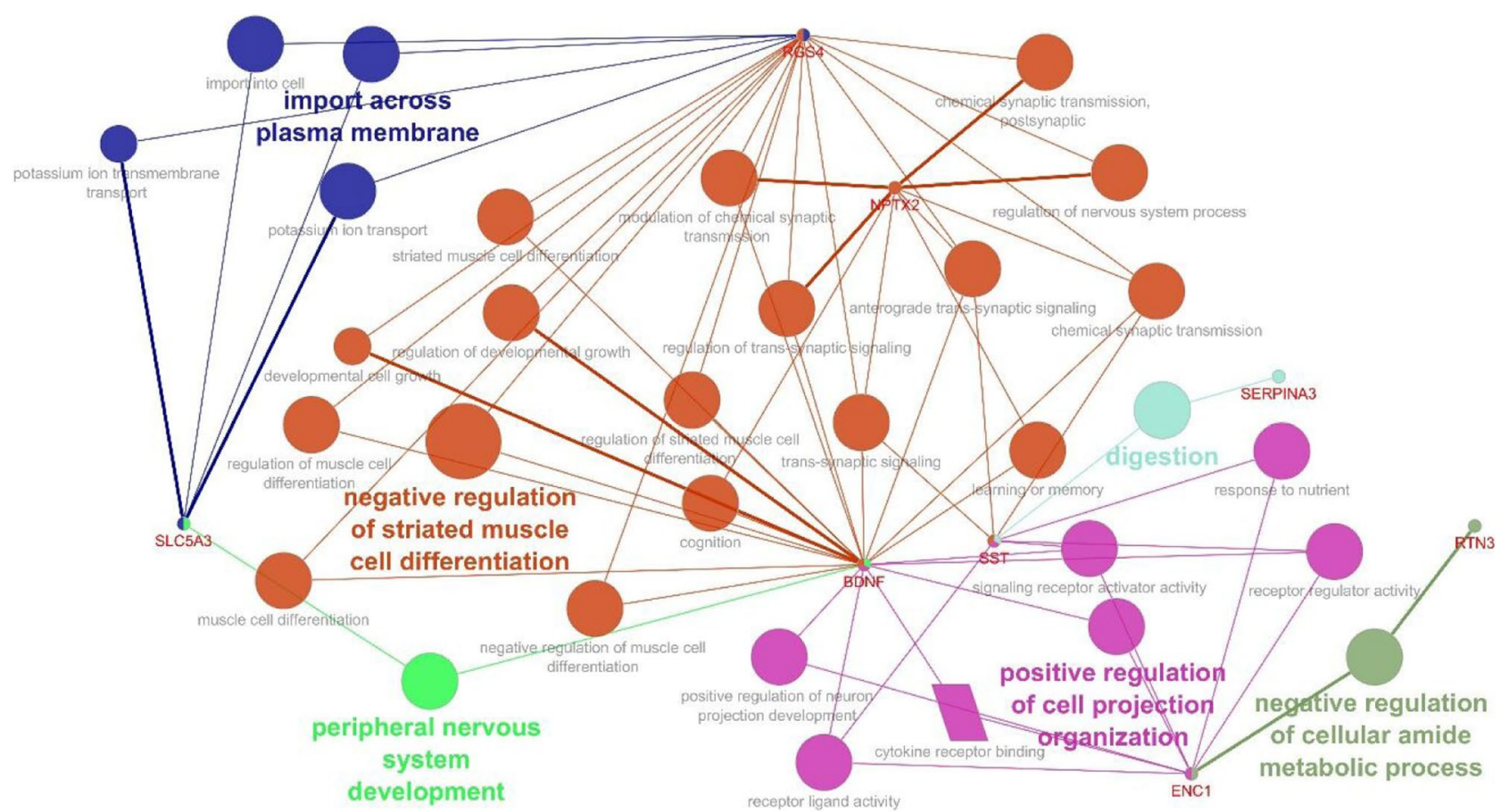

Fig. 6 Gene Ontology categories of common DEGs describing their physiological roles

(Saiz-Sanchez et al. 2010; Beal et al. 1985). In concordance with the above studies, our analysis found downregulation of SST receptors.
SERPINA3 or AACT is a 55-68 $\mathrm{kDa}$ serine protease inhibitor secreted by ependymal cells of the choroid plexus (Zhang and Janciauskiene 2002). Our PPI analysis identified

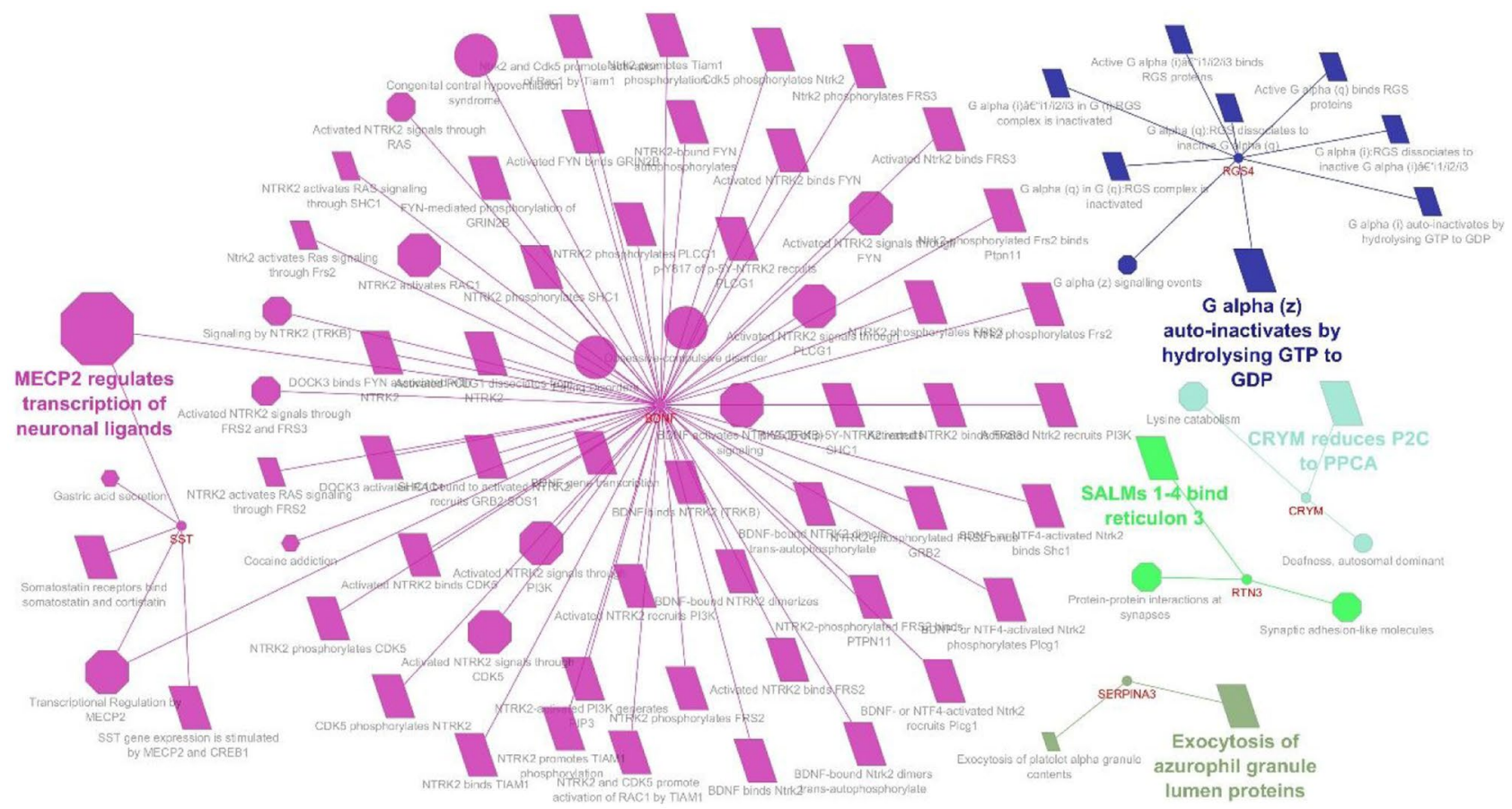

Fig. 7 Significant KEGG pathways of common DEGs 


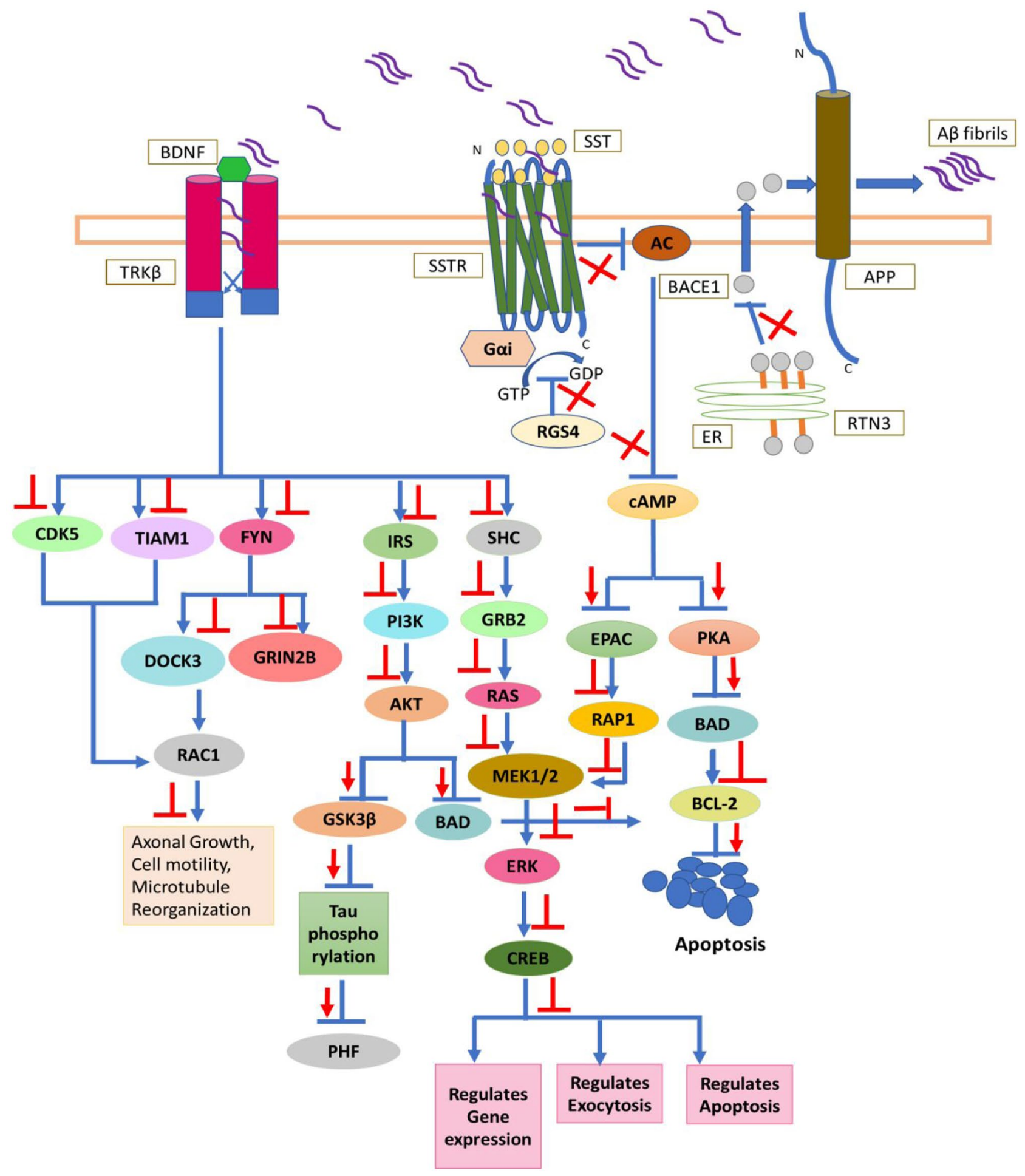

Fig. 8 Signaling mechanisms and cross-talk pathways underlying AD progression 
its interaction with APP, APOE and APOA1. Functional enrichment analysis revealed its role in digestion and exocytosis. In $\mathrm{AD}$, it was reported to be colocalized with amyloid plaques. The hydrophobic domain at the C-terminal of this enzyme interacts and forms a complex with amyloid fibrils. These complexes are known to upregulate SERPINA3, resulting in disruption of cognitive function (Abraham and Potter 1989; Eriksson et al. 1995). Apart from interacting with $\mathrm{A} \beta$ fibrils, it is also known to promote tau phosphorylation at Ser202, Thr231, Ser396 and Thr404 by augmenting extracellular signal-related kinase (ERK), glycogen synthase kinase-3 $\beta$ (GSK-3ß) and c-Jun N-terminal kinase (JNK), leading to inflammatory responses promoting neuronal death and degeneration (Tyagi et al. 2013; Padmanabhan et al. 2006).

RTN3, a transmembrane endoplasmic reticulum (ER) protein, belongs to a family of reticulons. Reticulons consist of four mammalian paralogs, i.e., RTN1, RTN2, RTN3 and RTN4, of which RTN3 and RTN4 are neuronal-specific. The members of this reticulon family possess a conserved QID triplet region, known as a reticulon homology domain (RHD) in their C-terminal region. This RHD domain was found to interact with the C-terminal domain of BACE1, which is involved in the formation of $A \beta$ peptides (Kume et al. 2009; He et al. 2006, 2007). The BACE1-RTN3 complex is reported to halt the axonal transport and enzymatic activity of BACE1 on APP, thereby terminating the amyloidogenic pathway. It was also reported that BACE1 was found to specifically interact with monomeric RTN3 rather than dimeric forms (Sharoar and Yan 2017; He et al. 2006). The formation of RTN3 aggregates was found to be regulated by B-cell receptor-associated protein 31 (BAP31), an integral ER membrane protein. Silencing of this gene leads to formation of RTN3 aggregates, thereby reducing the interaction with BACE1 which promotes A $\beta$ formation (He et al. 2004; Wang et al. 2019). Our functional enrichment analysis revealed the interactions of RTN3 with synaptic proteins and gene expression analysis demonstrated downregulation of this gene.

RGS4, a member of the RGS family, modulates G protein signaling activity by inhibiting $\mathrm{AC}$ and phospholipase $\mathrm{C}$ (PLC) activity. RGS4 inhibits G protein-coupled receptor (GPCR)-mediated APP cleavage, while downregulation of RGS4 enhances APP cleavage (Emilsson 2005). Functional enrichment analysis revealed that RGS4 was involved in various regulatory functions including modulation of chemical synaptic transmission, regulation of trans-synaptic signaling, nervous processes, striated muscle cell differentiation and regulation of cell growth. KEGG analysis revealed that active $\mathrm{G}$ alpha (i), (q) and (z) are binding partners of RGS4. Our gene expression analysis revealed downregulation of RGS4 in AD cases.

In summary, from the analysis, $B D N F, S S T$, SERPINA3, RTN3 and RGS4 were found to be crucially involved in AD pathogenesis. BDNF and $S S T$ trigger various signaling mechanisms including PKA, PI3K and AKT, which in turn inhibit GSK3 $\beta$ and BAD activity. This process results in the inhibition of apoptosis and promotion of neuronal growth. On the other hand, downregulation of BDNF and SST enables A $\beta$ fibrils to inhibit the aforementioned signaling mechanisms, thereby resulting in enhanced apoptosis and neuronal cell death. RTN3 interacts with BACE1 directly and impedes its access to APP cleavage, thereby promoting the nonamyloidogenic pathway. RGS4 acts in similar fashion as SST by hindering GTP hydrolysis (Fig. 8). The presence of $A \beta$ fibrils leads to AD progression; however, the aforesaid targets are believed to have substantial potential to counteract $A \beta$ toxicity.

Blue arrows represent signaling mechanisms in the absence of $A \beta$ fibrils, and red arrows represent signaling responses in the presence of $A \beta$ fibrils. BDNF: brainderived neurotrophic factor, TRK $\beta$ : tyrosine kinase $\beta$, SST: somatostatin, SSTR: somatostatin receptor, APP: amyloid precursor protein, AC: adenylyl cyclase, BACE1: betasecretase 1, ER: endoplasmic reticulum, RTN3: reticulon 3, GTP: guanosine triphosphate, GDP: guanosine diphosphate, RGS4: regulator of G protein signaling 4, cAMP: cyclic adenosine monophosphate, CDK5: cyclin-dependent kinase 5, TIAM1: T-lymphoma invasion and metastasis-inducing protein 1, FYN: Fyn kinase, IRS: insulin receptor substrate, AQ11SHC: src homology and collagen, DOCK3: dedicator of cytokinesis 3, GRIN2B: glutamate ionotropic receptor NMDA type subunit 2B, RAC1: Rac family small GTPase 1, PI3K: phosphatidylinositol-4,5-bisphosphate 3-kinase, AKT: AKT serine/threonine kinase, GSK3 $\beta$ : glycogen synthase kinase $3 \beta$, BAD:BCL2-associated agonist of cell death, GRB2: growth factor receptor bound-protein 2, RAS: KRAS proto-oncogene, GTPase, MEK: mitogen-activated protein kinase, ERK: extracellular signal-regulated kinase, CREB: cAMP responsive element binding protein 1, PHF: paired helical filaments, EPAC: Rap guanosine nucleotide exchange factor 3, RAP1: member of Ras oncogene family, PKA: protein kinase A, BCL2: BCL2 apoptosis regulator.

\section{Conclusion}

Systematic analysis of the metadata by considering all AD-related genetic datasets with a developed set of filtering criteria improved the precision of results. Through this analysis, SLC5A3, BDNF, SST, SERPINA3, RTN3, RGS4, $N P T X, E N C 1$ and $C R Y M$ were identified as potential genes involved in $\mathrm{AD}$ pathogenesis. Among the identified genes, $B D N F, S S T, S E R P I N A 3, R T N 3$ and RGS4 exhibited significant interactions with LDGs, and thus they were considered to play a major role in $\mathrm{AD}$ progression. 
List of Abbreviations A $\beta$ : Amyloid beta plaques; AC: Adenylylcycle; AD: Alzheimer's disease; APP: Amyloid precursor protein; ARDSI: Alzheimer's and related disorders society of India; BACE_1: Beta-secretase 1; BAP31: B-cell receptor-associated protein 31; BDNF: Brain-derived neurotrophic factor; CDK5: Cyclin-dependent kinase 5; COVID-19: Coronavirus disease 2019; CREB1: c-AMPresponsive element-binding protein 1; CRYM: Crystallin $\mathrm{Mu}$; DEGs: Differentially expressed genes; ENC1: Ectodermal-neural cortex 1; ERK: Extracellular signal-regulated kinase; FC: Fold change; FDR: False discovery rate; FRS2: Fibroblast growth factor receptor substrate 2; GEO: Gene Expression Omnibus; GDP: Guanosine diphosphate; GTP: Guanosine triphosphate; GRIN2B: Glutamate ionotropic receptor NMDA type subunit $2 \mathrm{~B}$; GSK-3 $\beta$ : Glycogen synthase kinase-3 $\beta$; HGCN: HUGO Gene Nomenclature Committee; JNK: c-Jun $\mathrm{N}$-terminal kinase; LDGs: Literature-derived genes; MAPT: Microtubule-associated protein tau; MECP2: Methyl-CpG binding protein 2; NCBI: National Center for Biotechnology Information; NFT: Neurofibrillary tangles; NPTX2: Neuronal pentraxin 2; PI3K: Phosphoinositide 3-kinase; PLC: Phospholipase C; PLCG1: Phospholipase C-gamma 1; PPI: Protein-protein interaction; PSEN1: Presenilin 1; RAS: Rat sarcoma; RGS4: Regulator of Gprotein signaling 4; RHD: Reticulon homology domain; SALM14: Synaptic adhesion-like molecule 1-4; SERPINA3: Serpin family A member 3; SHC1: Src homology 2 domain-containing 1; SLC5A3: Solute carrier family 5 member 3; SST: Somatostatin; STRING: Search tool for the retrieval of interacting genes/proteins; TIAM1: TLymphoma invasion and metastasis-inducing protein 1; TRK $\beta$ : Tyrosine kinase beta; US-FDA: US Food and Drug Administration

Acknowledgements We thank the Pharmacological Modelling and Simulation Centre (PMSC) and members of M.S. Ramaiah University of Applied Sciences, Bangalore, for their support throughout the work.

Authors' Contributions GNS analyzed the data and drafted the manuscript. GRS and R Burri supervised the work and finalized the manuscript.

\section{Declarations}

\section{Ethics Approval and Consent to Participate NA}

\section{Consent for Publication NA}

Competing Interests The authors declare that they have no competing interests.

\section{References}

Abraham CR, Potter H (1989) The protease inhibitor, $\alpha 1$-antichymotrypsin, is a component of the brain amyloid deposits in normal aging and Alzheimer's disease. Ann Med 21:77-81. https://doi.org/10.3109/07853898909149188

Aguado-Llera D, Canelles S, Frago LM et al (2018) The Protective Effects of IGF-I against $\beta$-Amyloid-related Downregulation of Hippocampal Somatostatinergic System Involve Activation of Akt and Protein Kinase A. Neuroscience 374:104-118. https:// doi.org/10.1016/j.neuroscience.2018.01.041

Akhtar A, Dhaliwal J, Saroj P et al (2020) Chromium picolinate attenuates cognitive deficit in ICV-STZ rat paradigm of sporadic Alzheimer's-like dementia via targeting neuroinflammatory and
IRS-1/PI3K/AKT/GSK-3 $\beta$ pathway. Inflammopharmacology 28:385-400. https://doi.org/10.1007/s10787-019-00681-7

Alzheimer's Association (2017) FDA-approved treatments for Alzheimer's. $1-5$

Alzheimer's disease facts and figures (2021). 2021 Alzheimer's disease facts and figures. Alzheimer's Dement 17:327-406. https://doi. org/10.1002/alz.12328

Andreoli V, De Marco EV, Trecroci F et al (2013) Potential involvement of GRIN2B encoding the NMDA receptor subunit NR2B in the spectrum of Alzheimer's disease. J Neural Transm 121:533542. https://doi.org/10.1007/s00702-013-1125-7

Astarita G, Jung KM, Berchtold NC et al (2010) Deficient liver biosynthesis of docosahexaenoic acid correlates with cognitive impairment in Alzheimer's disease. PLoS One 5:1-8. https://doi.org/10. 1371/journal.pone.0012538

Barrett T, Wilhite SE, Ledoux P et al (2013) NCBI GEO: archive for functional genomics data sets-update. Nucleic Acids Res 41:D991D995. https://doi.org/10.1093/nar/gks1193

Beal MF, Mazurek MF, Tran VT et al (1985) Reduced numbers of somatostatin receptors in the cerebral cortex in Alzheimer's disease. Science 229:289-291. https://doi.org/10.1126/science. 2861661

Berchtold NC, Coleman PD, Cribbs DH et al (2013) Synaptic genes are extensively downregulated across multiple brain regions in normal human aging and Alzheimer's disease. Neurobiol Aging 34:1653-1661. https://doi.org/10.1016/j.neurobiolaging.2012.11. 024

Bergen AA, Kaing S, Brinkten JB et al (2015) Gene expression and functional annotation of human choroid plexus epithelium failure in Alzheimer's disease. BMC Genomics 16. https://doi.org/10. 1186/s12864-015-2159-z

Berson A, Barbash S, Shaltiel G et al (2012) Cholinergic-associated loss of hnRNP-A/B in Alzheimer's disease impairs cortical splicing and cognitive function in mice. EMBO Mol Med 4:730-742. https://doi.org/10.1002/emmm.201100995

Bindea G, Mlecnik B, Hackl H et al (2009) ClueGO: a Cytoscape plugin to decipher functionally grouped gene ontology and pathway annotation networks. Bioinformatics 25:1091-1093. https://doi. org/10.1093/bioinformatics/btp101

Blair LJ, Nordhues BA, Hill SE et al (2013) Accelerated neurodegeneration through chaperone-mediated oligomerization of tau. J Clin Invest 123:4158-4169. https://doi.org/10.1172/JCI69003

Blalock EM, Buechel HM, Popovic J et al (2011) Microarray analyses of laser-captured hippocampus reveal distinct gray and white matter signatures associated with incipient Alzheimer's disease. J Chem Neuroanat 42:118-126. https://doi.org/10.1016/j.jchemneu.2011. 06.007

Blalock EM, Geddes JW, Chen KC et al (2004) Incipient Alzheimer's disease: Microarray correlation analyses reveal major transcriptional and tumor suppressor responses. Proc Natl Acad Sci U S A 101:2173-2178. https://doi.org/10.1073/pnas.0308512100

Brown GR, Hem V, Katz KS et al (2015) Gene: a gene-centered information resource at NCBI. Nucleic Acids Res 43:D36-D42. https:// doi.org/10.1093/nar/gku1055

Burgos-Ramos E, Hervás-Aguilar A, Aguado-Llera D et al (2008) Somatostatin and Alzheimer's disease. Mol Cell Endocrinol 286:104-111. https://doi.org/10.1016/j.mce.2008.01.014

Chahrour M, Sung YJ, Shaw C et al (2008) MeCP2, a key contributor to neurological disease, activates and represses transcription. Science 320:1224-1229. https://doi.org/10.1126/science.1153252

Den CK, Chang PT, Ping YH et al (2011) Gene expression profiling of peripheral blood leukocytes identifies and validates ABCB1 as a novel biomarker for Alzheimer's disease. Neurobiol Dis 43:698705. https://doi.org/10.1016/j.nbd.2011.05.023 
Chouraki V, Seshadri S (2014) Genetics of Alzheimer's disease. Elsevier

Cribbs DH, Berchtold NC, Perreau V et al (2012) Extensive innate immune gene activation accompanies brain aging, increasing vulnerability to cognitive decline and neurodegeneration: A microarray study. J Neuroinflammation 9. https://doi.org/10.1186/ 1742-2094-9-179

Deane R, Du YS, Submamaryan RK et al (2003) RAGE mediates amyloid- $\beta$ peptide transport across the blood-brain barrier and accumulation in brain. Nat Med 9:907-913. https://doi.org/10. 1038/nm890

Dunckley T, Beach TG, Ramsey KE et al (2006) Gene expression correlates of neurofibrillary tangles in Alzheimer's disease. Neurobiol Aging 27:1359-1371. https://doi.org/10.1016/j.neurobiolaging. 2005.08.013

Durrenberger PF, Fernando FS, Kashefi SN et al (2015) Common mechanisms in neurodegeneration and neuroinflammation: a BrainNet Europe gene expression microarray study. J Neural Transm 122:1055-1068. https://doi.org/10.1007/ s00702-014-1293-0

Durrenberger PF, Fernando FS, Magliozzi R et al (2012) Selection of novel reference genes for use in the human central nervous system: A BrainNet Europe Study. Acta Neuropathol 124:893-903. https://doi.org/10.1007/s00401-012-1027-z

Emilsson L (2005) Detection of differentially expressed genes in Alzheimer's disease. Uppsala University

Eriksson S, Janciauskiene S, Lannfelt L (1995) $\alpha 1$-Antichymotrypsin regulates Alzheimer $\beta$-amyloid peptide fibril formation. Proc Natl Acad Sci U S A 92:2313-2317. https://doi.org/10.1073/pnas.92.6. 2313

Fischer MT, Wimmer I, Höftberger R et al (2013) Disease-specific molecular events in cortical multiple sclerosis lesions. Brain 136:1799-1815. https://doi.org/10.1093/brain/awt110

Fitz NF, Tapias V, Cronican AA et al (2015) Opposing effects of Apoe / Apoal double deletion on amyloid- $\beta$ pathology and cognitive performance in APP mice. Brain 138:3699-3715. https://doi.org/ 10.1093/brain/awv293

He W, Hu X, Shi Q et al (2006) Mapping of Interaction Domains Mediating Binding between BACE1 and RTN/Nogo Proteins. J Mol Biol 363:625-634. https://doi.org/10.1016/j.jmb.2006.07.094

He W, Lu Y, Qahwash I et al (2004) Reticulon family members modulate BACE1 activity and amyloid- $\beta$ peptide generation. Nat Med 10:959-965. https://doi.org/10.1038/nm1088

He W, Shi Q, Hu X, Yan R (2007) The membrane topology of RTN3 and its effect on binding of RTN3 to BACE1. J Biol Chem 282:29144-29151. https://doi.org/10.1074/jbc.M704181200

Heinzen EL, Yoon W, Weale ME et al (2007) Alternative ion channel splicing in mesial temporal lobe epilepsy and Alzheimer's disease. Genome Biol 8. https://doi.org/10.1186/gb-2007-8-3-r32

Hokama M, Oka S, Leon J et al (2014) Altered expression of diabetesrelated genes in Alzheimer's disease brains: The Hisayama study. Cereb Cortex 24:2476-2488. https://doi.org/10.1093/cercor/ bht101

International D World Alzheimer Report (2018) The state of the art of dementia research: New frontiers. World Alzheimer Report 2018

Jha NK, Jha SK, Kumar D et al (2015) Impact of insulin degrading enzyme and neprilysin in Alzheimer's disease biology: Characterization of putative cognates for therapeutic applications. J Alzheimer's Dis 48:891-917. https://doi.org/10.3233/JAD-150379

Kang T, Qu Q, Xie Z, Cao B (2020) NDRG4 Alleviates A $\beta 1-40$ Induction of SH-SY5Y Cell Injury via Activation of BDNF-Inducing Signalling Pathways. Neurochem Res 45:1492-1499. https://doi. org/10.1007/s11064-020-03011-4

Kant S, Stopa EG, Johanson CE et al (2018) Choroid plexus genes for CSF production and brain homeostasis are altered in Alzheimer's disease. Fluids Barriers CNS 15. https://doi.org/10.1186/ s12987-018-0120-7

Kimura A, Hata S, Suzuki T (2016) Alternative Selection of $\beta$-Site APP-Cleaving Enzyme 1 (BACE1) Cleavage Sites in Amyloid $\beta$-Protein Precursor (APP) Harboring Protective and Pathogenic Mutations within the A $\beta$ Sequence. J Biol Chem 291:2404124053. https://doi.org/10.1074/jbc.M116.744722

Kumar CTS, Shaji KS, Varghese M NM (Eds) (2020) Dementiain India 2020 2. Alzheimer's and Related Disorders Society of India (ARDSI), Cochin

Kume H, Konishi Y, Murayama KS et al (2009) Expression of reticulon 3 in Alzheimer's disease brain. Neuropathol Appl Neurobiol 35:178-188. https://doi.org/10.1111/j.1365-2990.2008.00974.x

Lachen-Montes M, Zelaya MV, Segura V et al (2017) Progressive modulation of the human olfactory bulb transcriptome during Alzheimer's disease evolution: Novel insights into the olfactory signaling across proteinopathies. Oncotarget 8:69663-69679. https://doi.org/10.18632/oncotarget.18193

Lai MKP, Esiri MM, Tan MGK (2014) Genome-wide profiling of alternative splicing in Alzheimer's disease. Genomics Data 2:290-292. https://doi.org/10.1016/j.gdata.2014.09.002

Liang WS, Dunckley T, Beach TG et al (2007) Gene expression profiles in anatomically and functionally distinct regions of the normal aged human brain. Physiol Genomics 28:311-322. https://doi.org/ 10.1152/physiolgenomics.00208.2006

Liang WS, Dunckley T, Beach TG et al (2008a) Altered neuronal gene expression in brain regions differentially affected by Alzheimer's disease: A reference data set. Physiol Genomics 33:240-256. https://doi.org/10.1152/physiolgenomics.00242.2007

Liang WS, Reiman EM, Valla J et al (2008b) Alzheimer's disease is associated with reduced expression of energy metabolism genes in posterior cingulate neurons. Proc Natl Acad Sci USA 105:44414446. https://doi.org/10.1073/pnas.0709259105

Mackin RS, Insel P, Zhang J et al (2015) Cerebrospinal fluid $\alpha$-synuclein and Lewy body-like symptoms in normal controls, mild cognitive impairment, and Alzheimer's disease. J Alzheimer's Dis 43:1007-1016. https://doi.org/10.3233/JAD-141287

Maes OC, Schipper HM, Chertkow HM, Wang E (2009) Methodology for discovery of Alzheimer's disease blood-based biomarkers. Journals Gerontol - Ser A Biol Sci Med Sci 64:636-645. https:// doi.org/10.1093/gerona/glp045

Maes OC, Schipper HM, Chong G et al (2010) A GSTM3 polymorphism associated with an etiopathogenetic mechanism in Alzheimer disease. Neurobiol Aging 31:34-45. https://doi.org/10.1016/j. neurobiolaging.2008.03.007

Maes OC, Xu S, Yu B et al (2007) Transcriptional profiling of Alzheimer blood mononuclear cells by microarray. Neurobiol Aging 28:1795-1809. https://doi.org/10.1016/j.neurobiola ging.2006.08.004

Majores M, Kölsch H, Bagli M et al (2002) The insulin gene VNTR polymorphism in Alzheimer's disease: results of a pilot study. J Neural Transm 109:1029-1034. https://doi.org/10.1007/s007020200086

McKay EC, Beck JS, Khoo SK et al (2019) Peri-infarct upregulation of the oxytocin receptor in vascular dementia. J Neuropathol Exp Neurol 78:436-452. https://doi.org/10.1093/jnen/nlz023

Miller JA, Woltjer RL, Goodenbour JM et al (2013) Genes and pathways underlying regional and cell type changes in Alzheimer's disease. Genome Med 5. https://doi.org/10.1186/gm452

Miyashita A, Hatsuta H, Kikuchi M et al (2014) Genes associated with the progression of neurofibrillary tangles in Alzheimer's disease. Transl Psychiatry 4. https://doi.org/10.1038/tp.2014.35

Narayanan M, Huynh JL, Wang K, et al (2014) Common dysregulation network in the human prefrontal cortex underlies two neurodegenerative diseases. Mol Syst Biol 10:743. https://doi.org/10.15252/ msb.20145304 
Naughton BJ, Duncan FJ, Murrey DA et al (2014) Blood genome-wide transcriptional profiles reflect broad molecular impairments and strong blood-brain links in Alzheimer's disease. J Alzheimer's Dis 43:93-108. https://doi.org/10.3233/JAD-140606

Nho K, Kim S, Horgusluoglu E et al (2017) Association analysis of rare variants near the APOE region with CSF and neuroimaging biomarkers of Alzheimer's disease. BMC Med Genomics 10:29. https://doi.org/10.1186/s12920-017-0267-0

Nunez-Iglesias J, Liu CC, Morgan TE et al (2010) Joint genomewide profiling of miRNA and mRNA expression in Alzheimer's disease cortex reveals altered miRNA regulation PLoS One 5.https://doi.org/10.1371/journal.pone.0008898

Padmanabhan J, Levy M, Dickson DW, Potter H (2006) Alpha1antichymotrypsin, an inflammatory protein overexpressed in Alzheimer's disease brain, induces tau phosphorylation in neurons. Brain 129:3020-3034. https://doi.org/10.1093/brain/ awl 255

Patel H, Hodges AK, Curtis C et al (2019) Transcriptomic analysis of probable asymptomatic and symptomatic alzheimer brains. Brain Behav Immun 80:644-656. https://doi.org/10.1016/j.bbi. 2019.05.009

Piras IS, Krate J, Delvaux E et al (2019) Transcriptome Changes in the Alzheimer's Disease Middle Temporal Gyrus: Importance of RNA Metabolism and Mitochondria-Associated Membrane Genes. J Alzheimer's Dis 70:691-713. https://doi.org/10.3233/ JAD-181113

Readhead B, Haure-Mirande JV, Funk CC et al (2018) Multiscale Analysis of Independent Alzheimer's Cohorts Finds Disruption of Molecular, Genetic, and Clinical Networks by Human Herpesvirus. Neuron 99:64-82.e7. https://doi.org/10.1016/j.neuron. 2018.05.023

Saiz-Sanchez D, Ubeda-Bañon I, de la Rosa-Prieto C et al (2010) Somatostatin, tau, and $\beta$-amyloid within the anterior olfactory nucleus in Alzheimer disease. Exp Neurol 223:347-350. https:// doi.org/10.1016/j.expneurol.2009.06.010

Sandoval K, Umbaugh D, House A et al (2019) Somatostatin Receptor Subtype-4 Regulates mRNA Expression of Amyloid-Beta Degrading Enzymes and Microglia Mediators of Phagocytosis in Brains of 3xTg-AD Mice. Neurochem Res 44:2670-2680. https://doi.org/ 10.1007/s11064-019-02890-6

Sassi C, Guerreiro R, Gibbs R et al (2014) Investigating the role of rare coding variability in Mendelian dementia genes (APP, PSEN1, PSEN2, GRN, MAPT, and PRNP) in late-onset Alzheimer's disease. Neurobiol Aging 35:2881.e1-2881.e6. https://doi.org/10. 1016/j.neurobiolaging.2014.06.002

Scacchi R, Gambina G, Moretto G, Corbo RM (2009) Variability of AChE, $\mathrm{BChE}$, and ChAT genes in the late-onset form of Alzheimer's disease and relationships with response to treatment with Donepezil and Rivastigmine. Am J Med Genet Part B Neuropsychiatr Genet 150B:502507. https://doi.org/10.1002/ajmg.b.30846

Schrötter A, Pfeiffer K, El Magraoui F et al (2012) The amyloid precursor protein (APP) family members are key players in S-adenosylmethionine formation by MAT2A and modify BACE1 and PSEN1 gene expressionrelevance for Alzheimer's disease. Mol Cell Proteomics 11:1274-1288. https://doi.org/10.1074/mcp.M112.019364

Sharoar MG, Yan R (2017) Effects of altered RTN3 expression on BACE1 activity and Alzheimer's neuritic plaques. Rev Neurosci 28:145-154. https://doi.org/10.1515/revneuro-2016-0054
Shinohara M, Tachibana M, Kanekiyo T, Bu G (2017) Role of LRP1 in the pathogenesis of Alzheimer's disease: evidence from clinical and preclinical studies. J Lipid Res 58:1267-1281. https://doi.org/ 10.1194/jlr.R075796

Silva ART, Grinberg LT, Farfel JM et al (2012) Transcriptional alterations related to neuropathology and clinical manifestation of Alzheimer's disease. PLoS One 7. https://doi.org/10.1371/journal. pone.0048751

Sood S, Gallagher IJ, Lunnon K et al (2015) A novel multi-tissue RNA diagnostic of healthy ageing relates to cognitive health status. Genome Biol 16. https://doi.org/10.1186/s13059-015-0750-x

Stopa EG, Tanis KQ, Miller MC et al (2018) Comparative transcriptomics of choroid plexus in Alzheimer's disease, frontotemporal dementia and Huntington's disease: Implications for CSF homeostasis. Fluids Barriers CNS 15.https://doi.org/10.1186/ s12987-018-0102-9

Tan MG, Chua WT, Esiri MM et al (2010) Genome wide profiling of altered gene expression in the neocortex of Alzheimer's disease. J Neurosci Res 88:1157-1169. https://doi.org/10. 1002/jnr.22290

Tyagi E, Fiorelli T, Norden M, Padmanabhan J (2013) Alpha 1-antichymotrypsin, an inflammatory protein overexpressed in the brains of patients with Alzheimer's disease, induces Tau hyperphosphorylation through c-Jun N-terminal kinase activation. Int J Alzheimers Dis 2013:1-12. https://doi.org/ $10.1155 / 2013 / 606083$

von Mering C, Huynen M, Jaeggi D et al (2003) STRING: a database of predicted functional associations between proteins. Nucleic Acids Res 31:258-261

Wang T, Chen J, Hou Y et al (2019) BAP31 deficiency contributes to the formation of amyloid-b plaques in Alzheimer's disease by reducing the stability of RTN3. FASEB J 33:4936-4946. https:// doi.org/10.1096/fj.201801702R

Webster JA, Gibbs JR, Clarke J et al (2009) Genetic Control of Human Brain Transcript Expression in Alzheimer Disease. Am J Hum Genet 84:445-458. https://doi.org/10.1016/j.ajhg.2009.03.011

Williams C, Shai RM, Wu Y et al (2009) Transcriptome analysis of synaptoneurosomes identifies neuroplasticity genes overexpressed in incipient Alzheimer's disease. PLoS One 4.https://doi.org/10. 1371/journal.pone.0004936

Wojsiat J, Laskowska-Kaszub K, Alquézar C et al (2017) Familial Alzheimer's Disease Lymphocytes Respond Differently Than Sporadic Cells to Oxidative Stress: Upregulated p53-p21 Signaling Linked with Presenilin 1 Mutants. Mol Neurobiol 54:5683-5698. https://doi.org/10.1007/s12035-016-0105-y

Zhang S, Janciauskiene S (2002) Multi-functional capability of proteins: $\alpha 1$-antichymotrypsin and the correlation with Alzheimer's disease. J Alzheimer's Dis 4:115-122. https://doi.org/10.3233/ JAD-2002-4206

Zhou J, Li X-M, Jiang T et al (2013) Lack of association between COMT Val158Met polymorphism and late-onset Alzheimer's disease in Han Chinese. Neurosci Lett 554:162-166. https://doi. org/10.1016/J.NEULET.2013.09.006

Publisher's Note Springer Nature remains neutral with regard to jurisdictional claims in published maps and institutional affiliations. 


\section{Authors and Affiliations}

\section{Hema Sree GNS ${ }^{1}$. Saraswathy Ganesan Rajalekshmi ${ }^{1,2}$ (D) Raghunadha R. Burri $^{3}$}

Hema Sree GNS

nagasai.hemasree615@gmail.com

Raghunadha R. Burri

rburri@gmail.com

1 Pharmacological Modelling and Simulation Centre, M. S.

Ramaiah University of Applied Sciences, New BEL Road,

Bangalore, India 560054
2 Department of Pharmacy Practice, Faculty of Pharmacy, M. S. Ramaiah University of Applied Sciences, New BEL Road, Bangalore, India 560054

3 Dr. Reddy's Laboratories, Hyderabad, India 500090 